Comment. Math. Helv. 73 (1998) 137-174

(C) 1998 Birkhäuser Verlag, Basel

0010-2571/98/010137-38 \$1.50+0.20/0

Commentarii Mathematici Helvetici

\title{
The behaviour at infinity of the Bruhat decomposition
}

Michel Brion

\begin{abstract}
For a connected reductive group $G$ and a Borel subgroup $B$, we study the closures of double classes $B g B$ in a $(G \times G)$-equivariant "regular" compactification of $G$. We show that these closures $\overline{B g B}$ intersect properly all $(G \times G)$-orbits, with multiplicity one, and we describe the intersections. Moreover, we show that almost all $\overline{B g B}$ are singular in codimension two exactly. We deduce this from more general results on $B$-orbits in a spherical homogeneous space $G / H$; they lead to formulas for homology classes of $H$-orbit closures in $G / B$, in terms of Schubert cycles.
\end{abstract}

Mathematics Subject Classification (1991). 14L30, 14M15, $14 \mathrm{M} 17$.

Keywords. Bruhat decomposition, regular embedding, Chow ring.

\section{Introduction}

Let $G$ be a connected complex reductive group, $B \subset G$ a Borel subgroup and $H \subset G$ a spherical subgroup, that is, the homogeneous space $G / H$ contains a dense $B$-orbit. Then any equivariant embedding $X$ of $G / H$ contains only finitely many $B$-orbits (see $[\mathrm{Kn}]$ for a simple proof of this result).

A natural question is to describe the $B$-orbit closures in a smooth complete embedding $X$ of $G / H$, and their classes in the Chow group $A_{*}(X)$; recall that $A_{*}(X)$ is then isomorphic to the integral homology of $X$, and is generated as a group by classes of $B$-orbit closures. Another, closely related question is to describe the $H$-orbit closures in the flag variety $G / B$, and their classes in $A_{*}(G / B)$.

A classical example is the case where $G / H$ is complete, that is, $H$ is a parabolic subgroup of $G$. Then the $B$-orbit closures in $G / H$ are the Schubert varieties, and their classes (the Schubert cycles) form a basis of the group $A_{*}(G / H)$.

In the present article, we obtain partial answers to our questions in the general setting of a spherical homogeneous space, and more precise results when the space is $G$ and the group is $G \times G$ acting on $G$ by left and right multiplication. In this case, the first question asks for the behaviour "at infinity" of the closures of $B$-double cosets in $G$.

To any $B$-orbit closure $Y$ in a spherical homogeneous space $G / H$, we associate 
a subset $W(Y)$ of the Weyl group of $G$, and a function $d(Y, \cdot)$ on $W(Y)$ with values in integral powers of 2 (see 1.1). Given a smooth complete embedding $X$ of $G / H$ which is regular in the sense of [BDP], and a closed $G$-orbit $Z \subset X$, we show that the closure of $Y$ in $X$ has proper intersection with $Z$. Moreover, the components of $Y \cap Z$ are the Schubert varieties in $Z$ parametrized by $W(Y)$, and the corresponding intersection multiplicities are the values of the function $d(Y, \cdot)$ (up to twists, see 1.4).

On the other hand, any $H$-orbit closure $V \subset G / B$ defines obviously a $B$-orbit closure $Y \subset G / H$; the decomposition of the class of $V$ in $A_{*}(G / B)$ on the basis of Schubert cycles turns out to be determined by $W(Y)$ and $d(Y, \cdot)$ (see 1.5).

In the case of the homogeneous space $G$ under $G \times G$, the function $d(Y, \cdot)$ has constant value 1 (see 2.1). It follows that all closures of $(B \times B)$-orbits in any regular completion $X$ of $G$ are smooth in codimension one (as shown by Barbasch and Evens, closures of $B$-orbits in spherical varieties can be singular in codimension one, see $[\mathrm{BE}])$.

Actually, any closure in $X$ of a $B$-double class in $G$ is singular in codimension two, apart from trivial exceptions (see 2.2). This uniform result contrasts with the situation for Schubert varieties, where the characterization of smoothness is quite delicate (see e.g. $[\mathrm{C}],[\mathrm{K}]$ and $[\mathrm{L}]$ ).

The behaviour "at infinity" of $(B \times B)$-orbit closures is described in 2.1, and the case of parabolic subgroups of $G$ is treated in more detail in 2.3. As an application, we construct a degeneration of the diagonal of a flag variety to a sum of Schubert cycles.

These results are then applied to the study of the Chow ring $A^{*}(X)$ where $X$ is a regular completion of $G$. For this, we use Edidin and Graham's equivariant intersection theory (see $[\mathrm{EG}]$ and also $[\mathrm{Br}]$ ); it could be replaced by equivariant cohomology but we prefer a purely algebraic approach. In 3.1 , we describe the equivariant Chow ring of $X$ in terms of the closed $(G \times G)$-orbits, generalizing results of Littelmann and Procesi (see $[\mathrm{LP}]$ ). Then we give closed formulae for the equivariant classes of $(B \times B)$-orbit closures (see 3.2$)$.

In the case where $X$ is the canonical regular completion of a semisimple adjoint group, we construct a basis of the Chow group of $X$ (see 3.3) and we determine the intersection numbers of any two $(B \times B)$-orbit closures of complementary dimensions (see 3.4). Our picture of the Chow ring confirms the idea that the geometry of regular completions of $G$ is governed by the closed $(G \times G)$-orbits and by the closure of a maximal torus, as shown by De Concini and Procesi (see [DP1] and [DP2]).

Using the general methods of Part 1, several results of the present work can be extended to other spherical homogenenous spaces, e.g. to split symmetric spaces; this will be developed elsewhere.

The structure results for regular group completions which are used in our article are gathered in an appendix. These results are due to DeConcini and Procesi in the case of a semisimple adjoint group and, more generally, of an adjoint sym- 
metric space (see [DP1]). For a connected reductive group, they can be deduced from embedding theory of spherical homogeneous spaces. Here we follow a direct, characteristic-free approach based on one-parameter subgroups. As a consequence, all results of the present work which concern regular completions of $G$ are valid in arbitrary characteristics, provided that each $(G \times G)$-orbit map is separable.

\section{Orbit closures of Borel subgroups in spherical varieties}

\subsection{Preliminaries}

We begin by fixing notation, defining the set $W(Y)$ and the function $d(Y, \cdot)$ and studying their first properties. Throughout the paper, we will use freely classical notions and results on the Bruhat decomposition in reductive groups and on the combinatorics of Weyl groups; for this, we refer to $[\mathrm{H}]$ and $[\mathrm{Sp}]$.

Let $G$ be a connected complex reductive group, $B \subset G$ a Borel subgroup, and $T \subset B$ a maximal torus of dimension $r$. Denote by $W$ the Weyl group and by $\Phi$ the root system of $(G, T)$. We have the subset $\Phi^{+}$of positive roots and its subset $\Delta$ of simple roots. For $\alpha \in \Delta$ we denote by $s_{\alpha} \in W$ the corresponding reflection and by $P_{\alpha}=B \cup B s_{\alpha} B$ the corresponding minimal parabolic subgroup. The length of $w \in W$ is denoted by $l(w)$, and the longest element of $W$ is denoted by $w_{0}$.

Let $P \supset B$ be a parabolic subgroup with Levi subgroup $L \supset T$. Denote by $W_{L}$ the Weyl group and by $\Phi_{L}$ the set of roots of $(L, T)$. Set

$$
W^{L}:=\left\{w \in W \mid l(w v)=l(w)+l(v) \forall v \in W_{L}\right\}=\left\{w \in W \mid w\left(\Phi_{L}^{+}\right) \subset \Phi^{+}\right\} .
$$

Then $W^{L}$ is a system of representatives of the quotient $W / W_{L}$; moreover, the unique element of maximal length in $W^{L}$ is $w_{0} w_{0, L}$ where $w_{0, L}$ denotes the longest element of $W_{L}$. The space $G / P$ is the disjoint union of the $B w P / P\left(w \in W^{L}\right)$. Moreover, the dimension of $\overline{B w P} / P$ is the length of $w$. Denoting by $B^{-}$the Borel subgroup of $G$ such that $B^{-} \cap B=T$ and by $Q \supset B^{-}$the parabolic subgroup opposed to $P$, we have $P \cap Q=L$. The length of $w \in W^{L}$ is the codimension of $\overline{B w Q} / Q$ in $G / Q$.

Consider now a variety $X$ with a $G$-action (by variety we mean a reduced and irreducible algebraic complex scheme, and by subvariety, a closed subscheme which is a variety). Following [Kn], the set of $B$-invariant subvarieties of $X$ is denoted by $\mathcal{B}(X)$. For $Y \in \mathcal{B}(X)$ and $w \in W$, the set $\overline{B w Y}$ is in $\mathcal{B}(X)$ (this set is denoted by $w * Y$ in $[\mathrm{Kn}]$, where the resulting operation on $\mathcal{B}(X)$ is studied). The map

$$
\begin{array}{ccc}
\overline{B w B} \times Y & \rightarrow \overline{B w Y} \\
(g, y) & \mapsto g y
\end{array}
$$

is invariant under the $B$-action defined by $b(g, y)=\left(g b^{-1}, b y\right)$. Denoting by $\overline{B w B} \times{ }_{B} Y$ the quotient, we obtain a map

$$
\pi_{Y, w}: \overline{B w B} \times_{B} Y \rightarrow \overline{B w Y} .
$$


Because $\overline{B w B} / B$ is complete, $\pi_{Y, w}$ is proper and hence surjective.

Definitions. Let $d(Y, w)$ be the degree of $\pi_{Y, w}$ if this map is generically finite; otherwise, set $d(Y, w)=0$.

Let $W(Y)$ be the set of all $w \in W$ such that $\pi_{Y, w}$ is generically finite and that $\overline{B w Y}$ is $G$-invariant.

Lemma. Let $Y \in \mathcal{B}(X)$.

(i) For any $\tau$ and $w$ in $W$ such that $l(\tau w)=l(\tau)+l(w)$, we have

$$
d(Y, \tau w)=d(Y, w) d(\overline{B w Y}, \tau) .
$$

(ii) For any $w \in W$ such that $\overline{B w Y}$ contains only finitely many B-orbits, the integer $d(Y, w)$ is 0 or a power of 2 .

(iii) For any $w \in W$ such that $d(Y, w) \neq 0$, we have

$$
W(\overline{B w Y})=\{\tau \in W \mid l(\tau w)=l(\tau)+l(w) \text { and } \tau w \in W(Y)\} .
$$

(iv) The set $W(Y)$ is not empty.

(v) If $X=G / P$ where $P \supset B$ is a parabolic subgroup with Levi subgroup $L \supset T$ and $Y=\overline{B w P} / P$ with $\tau \in W^{L}$, then $W(Y)=\left\{w_{0} w_{0, L} w^{-1}\right\}$. Moreover, we have $d\left(Y, w_{0} w_{0, L} w^{-1}\right)=1$.

Proof. (i) By the Bruhat decomposition, the canonical map

$$
\overline{B \tau B} \times_{B} \overline{B w B} \rightarrow \overline{B \tau w B}
$$

is birational. It follows that the degree of $\pi_{Y, \tau w}$ is equal to the degree of the map

$$
\overline{B \tau B} \times_{B} \overline{B w B} \times_{B} Y \rightarrow \overline{B \tau w Y} .
$$

But the latter factors as

$$
\overline{B \tau B} \times_{B} \overline{B w B} \times_{B} Y \rightarrow \overline{B \tau B} \times_{B} \overline{B w Y}
$$

of degree $d(Y, w)$, followed by

$$
\overline{B \tau B} \times_{B} \overline{B w Y} \rightarrow \overline{B \tau w Y}
$$

of degree $d(\overline{B w Y}, \tau)$.

(ii) Write $w=\tau s_{\alpha}$ where $\tau \in W, \alpha \in \Delta$ and $l(w)=l(\tau)+1$. Then $\overline{B s_{\alpha} Y} \subset$ $\overline{B w Y}$ and, by (i):

$$
d(Y, w)=d\left(Y, s_{\alpha}\right) d\left(\overline{B s_{\alpha} Y}, \tau\right) .
$$

By [RS] $\S 4$ or [Kn] 3.2 (see also [Br] 6.2), we have $d\left(Y, s_{\alpha}\right) \leq 2$. We conclude by induction over $l(w)$. 
(iii) If $\tau \in W(\overline{B w Y})$ then

$$
l(\tau)+l(w)=\operatorname{dim}(\overline{B \tau B w Y})-\operatorname{dim}(Y) \leq \operatorname{dim}(\overline{B \tau B w B} / B)
$$

which implies that $l(\tau)+l(w)=l(\tau w)$ and that $\overline{B \tau B w B}=\overline{B \tau w B}$. Therefore, $\tau w \in W(Y)$. The converse is similar.

(iv) We argue by induction over the codimension of $Y$ in $G Y$. If $Y=G Y$ then $W(Y)=\{1\}$. Otherwise we can find a minimal parabolic subgroup $P_{\alpha} \supset B$ such that $P_{\alpha} Y \neq Y$. Then $W\left(P_{\alpha} Y\right)$ is not empty, and we conclude by (iii).

(v) Because $w \in W^{L}$, we have $\overline{B w P}=\overline{B w w_{0, L} B}$. Let $\tau \in W(\overline{B w P} / P)$. Then the map

$$
\overline{B \tau B} \times_{B} \overline{B w w_{0, L} B} \rightarrow G
$$

is generically finite and surjective. By the Bruhat decomposition, this map is birational and $\tau=w_{0} w_{0, L} w^{-1}$.

Remark. If $X=G / Q$ with $Q \supset B^{-}$and $Y=\overline{B w Q} / Q$ with $w \in W^{L}$, then $W(Y)=\left\{w^{-1}\right\}$ and $d\left(Y, w^{-1}\right)=1$.

\subsection{Cancellative and induced actions}

This section contains technical results which will play a key role in our study of regular group completions.

Definition. The action of $G$ on a variety $X$ is cancellative if for any distinct $Y_{1}$, $Y_{2}$ in $\mathcal{B}(X)$ and for any $\alpha \in \Delta$ such that $P_{\alpha} Y_{1} \neq Y_{1}$ and $P_{\alpha} Y_{2} \neq Y_{2}$ we have $P_{\alpha} Y_{1} \neq P_{\alpha} Y_{2}$.

Equivalently, for any distinct $Y_{1}, Y_{2} \in \mathcal{B}(X)$ such that $G Y_{1}=G Y_{2}$, the sets $W\left(Y_{1}\right)$ and $W\left(Y_{2}\right)$ are disjoint. In particular, any $Y \in \mathcal{B}(X)$ is uniquely determined by $G Y$ and $W(Y)$.

For example, the $G$-action on $G / P$ is cancellative for any parabolic subgroup $P$ of $G$ (this follows e.g. from Lemma 1.1). The $(G \times G)$-action on $G$ by left and right multiplication is cancellative, too. But the diagonal action of $G=\mathrm{PGL}_{2}$ on $\mathbf{P}^{1} \times \mathbf{P}^{1}$ is not cancellative. Indeed, let $B$ be the standard Borel subgroup of $G$ and let $\infty$ be the $B$-fixed point in $\mathbf{P}^{1}$. Then $Y_{1}:=\mathbf{P}^{1} \times\{\infty\}$ and $Y_{2}:=\{\infty\} \times \mathbf{P}^{1}$ are $B$-invariant subvarieties with $Y_{1} \neq G Y_{1}=G Y_{2} \neq Y_{2}$.

Definition. Let $P \supset B$ be a parabolic subgroup with Levi subgroup $L \supset T$ and let $X^{\prime}$ be a $L$-variety. The induced variety $X$ is the quotient of $G \times X^{\prime}$ by the diagonal $P$-action where $P$ acts on $G$ by right multiplication, and on $X^{\prime}$ through its quotient group $L$. We denote $X$ by $G \times{ }_{P} X^{\prime}$ and we identify $X^{\prime}$ to the $P$-invariant subvariety $P \times_{P} X^{\prime} \subset X$, the fiber at $P / P$ of the canonical map $p: G \times{ }_{P} X^{\prime} \rightarrow G / P$. 
Lemma. Notation being as above, any $Y \in \mathcal{B}(X)$ can be written uniquely as $\overline{B w Y^{\prime}}$ where $w \in W^{L}$ and $Y^{\prime} \subset X^{\prime}$ is a $(B \cap L)$-invariant subvariety. Then

$$
W(Y)=\left\{\tau \in W \mid \tau w \in w_{0} w_{0, L} W_{L}\left(Y^{\prime}\right) \text { and } l(\tau)=\operatorname{codim}_{G Y}(Y)\right\}
$$

and for any $w \in W(Y)$, we have

$$
d(Y, w)=d_{L}\left(Y^{\prime}, w_{0, L} w_{0} \tau w\right) .
$$

Furthermore, the $G$-action on $X$ is cancellative if and only if the L-action on $X^{\prime}$ is.

Proof. Let $Y \in \mathcal{B}(X)$. Then there exists a unique $w \in W^{L}$ such that $B w P / P$ is dense in $p(Y)$. Moreover, $Y \cap p^{-1}(w P / P)$ is invariant under $B \cap w P w^{-1}$. This group contains $w(B \cap L) w^{-1}$ because $w$ is in $W^{L}$. Therefore, we have $Y \cap$ $p^{-1}(w P / P)=w Y^{\prime}$ for a unique $(B \cap L)$-invariant subvariety $Y^{\prime} \subset X^{\prime}$. It follows that $B w Y^{\prime}=Y \cap p^{-1}(B w P / P)$ is dense in $Y$.

For the second statement, consider first the case where $w=1$; then $Y=Y^{\prime}$. Let $\tau \in W\left(Y^{\prime}\right)$. Write $\tau=\tau^{L} \tau_{L}$ where $\tau^{L} \in W^{L}$ and $\tau_{L} \in W_{L}$. Because $\overline{B \tau Y^{\prime}}=G Y^{\prime}$, we must have $\tau^{L}=w_{0} w_{0, L}$ and $\tau_{L} \in W_{L}\left(Y^{\prime}\right)$. Therefore, $W\left(Y^{\prime}\right)=$ $w_{0} w_{0, L} W_{L}\left(Y^{\prime}\right)$. Moreover, $d(Y, \tau)=d_{L}\left(Y^{\prime}, \tau_{L}\right)$.

In the general case, it follows from Lemma 1.1 that $\tau \in W(Y)$ if and only if $l(\tau)=\operatorname{codim}_{G Y}(Y)$ and $\tau w \in W\left(Y^{\prime}\right)$. The latter amounts to: $\tau w=w_{0} w_{0, L} u$ for some $u \in W_{L}\left(Y^{\prime}\right)$. Because $w \in W^{L}$ we have $d\left(Y^{\prime}, w\right)=1$. Therefore, we have by Lemma 1.1: $d(Y, \tau)=d\left(Y^{\prime}, \tau w\right)=d_{L}\left(Y^{\prime}, u\right)$.

If the $G$-action on $X$ is cancellative, then it is easy to see that the $L$-action on $X^{\prime}$ is, too. For the converse, let $Y_{1}, Y_{2}$ be distinct $B$-invariant subvarieties of $X$ and let $\alpha \in \Delta$ such that $Y_{1} \neq P_{\alpha} Y_{1}=P_{\alpha} Y_{2} \neq Y_{2}$. For $i=1,2$, write $Y_{i}=\overline{B w_{i} Y_{i}^{\prime}}$ as above. Then $P_{\alpha} Y_{i}=\overline{B s_{\alpha} w_{i} Y_{i}^{\prime}}$ because $P_{\alpha} Y_{i} \neq Y_{i}$. We distinguish between three cases.

(i) $s_{\alpha} w_{1} \notin W^{L}$ and $s_{\alpha} w_{2} \notin W^{L}$. Then $s_{\alpha} w_{1}\left(\beta_{1}\right) \notin \Phi^{+}$for some simple root $\beta_{1}$ of $(L, T)$. It follows that $w_{1}\left(\beta_{1}\right)=\alpha$ and that $s_{\alpha} w_{1}=w_{1} s_{\beta_{1}}$. So $s_{\beta_{1}} Y_{1}^{\prime} \neq Y_{1}^{\prime}$ (because $P_{\alpha} Y_{1} \neq Y_{1}$ ) and $P_{\alpha} Y_{1}=\overline{B w_{1} s_{\beta_{1}} Y_{1}^{\prime}}$. Similarly, $s_{\alpha} w_{2}=w_{2} s_{\beta_{2}}$ for some simple root $\beta_{2}$ of $(L, T)$. Therefore, $w_{1}=w_{2}$ and $P_{\beta_{1}} Y_{1}^{\prime}=P_{\beta_{2}} Y_{2}^{\prime}$. Because the $L$-action on $X^{\prime}$ is assumed to be cancellative, this implies $Y_{1}^{\prime}=Y_{2}^{\prime}$.

(ii) $s_{\alpha} w_{1} \in W^{L}$ and $s_{\alpha} w_{2} \in W^{L}$. Then $s_{\alpha} w_{1}=s_{\alpha} w_{2}$ whence $w_{1}=w_{2}$, and $Y_{1}^{\prime}=Y_{2}^{\prime}$.

(iii) $s_{\alpha} w_{1} \notin W^{L}$ and $s_{\alpha} w_{2} \in W^{L}$. Write $s_{\alpha} w_{1}=w_{1} s_{\beta_{1}}$ as in case (i). Then $\overline{B w_{1} s_{\beta_{1}} Y_{1}^{\prime}}=\overline{B s_{\alpha} w_{2} Y_{2}^{\prime}}$ whence $w_{1}=s_{\alpha} w_{2}$. Therefore, $s_{\alpha} w_{1}=w_{2} \in W^{L}$, a contradiction.

Remark. Let $Q \supset B^{-}$be the parabolic subgroup opposite to $P$. Consider the induced variety $G \times{ }_{Q} X^{\prime}$. Then, for $w \in W^{L}$ and $Y^{\prime} \in \mathcal{B}\left(X^{\prime}\right)$, we have

$$
W\left(\overline{B w Y^{\prime}}\right)=\left\{\tau \in W \mid \tau w \in W_{L}\left(Y^{\prime}\right) \text { and } l(\tau)=\operatorname{codim}_{G Y^{\prime}}\left(\overline{B w Y^{\prime}}\right)\right\}
$$


and $d\left(\overline{B w Y^{\prime}}, \tau\right)=d_{L}\left(Y^{\prime}, \tau w\right)$ whenever $\tau \in W\left(\overline{B w Y^{\prime}}\right)$. Thus, the formulation of the Lemma above is much simpler; note however that $Y^{\prime}$ (viewed as a subvariety of $\left.G \times{ }_{Q} X^{\prime}\right)$ is not $B$-invariant .

\subsection{Intersection multiplicities of invariant subvarieties}

In this section, we give a geometric interpretation of $W(Y)$ and $d(Y, w)$.

Let $X$ be a complete, non-singular $G$-variety, let $Y \subset X$ be a $B$-invariant subvariety such that $G Y=X$, and let $Z \subset X$ be a $G$-invariant subvariety. We denote by $\mathcal{C}(Y \cap Z)$ the set of irreducible components of the intersection of $Y$ and $Z$. Recall that each $C \in \mathcal{C}(Y \cap Z)$ satisfies $\operatorname{dim}(C) \geq \operatorname{dim}(Y)+\operatorname{dim}(Z)-\operatorname{dim}(X)$. By definition, $Y$ and $Z$ meet properly along $C$ if equality holds above, or equivalently if $\operatorname{codim}_{Z}(C)=\operatorname{codim}_{X}(Y)$. In this case, we denote by $i(C, Y \cdot Z)$ the intersection multiplicity of $Y$ and $Z$ along $C$, see [F] Chap. 7.

Lemma. (i) If $Y$ meets $Z$ properly, then

$$
W(Y) \subset \bigcup_{C \in \mathcal{C}(Y \cap Z)} W(C) .
$$

If moreover $Y$ meets properly any $G$-invariant subvariety of $Z$, then equality holds above, and $G C=Z$ for any $C \in \mathcal{C}(Y \cap Z)$.

(ii) Assume that $Y$ meets properly any $G$-invariant subvariety of $Z$, and that the $G$-action on $Z$ is cancellative. Then $\mathcal{C}(Y \cap Z)$ is the set of all $C \in \mathcal{B}(Z)$ such that $W(C)$ meets $W(Y)$. Moreover, $W(Y)$ is the disjoint union of the $W(C)$ for $C \in \mathcal{C}(Y \cap Z)$. Finally, we have for any $C \in \mathcal{C}(Y \cap Z)$ :

$$
d(C, w) i(C, Y \cdot Z)=d(Y, w)
$$

Proof. (i) Assume that $Y$ meets $Z$ properly. Let $w \in W(Y)$. The generically finite, surjective morphism

$$
\pi_{Y, w}: \overline{B w B} \times_{B} Y \rightarrow X
$$

restricts to a surjective morphism $\overline{B w B} \times_{B}(Y \cap Z) \rightarrow Z$. Thus, there exists $C \in \mathcal{C}(Y \cap Z)$ such that the morphism

$$
\pi_{C, w}: \overline{B w B} \times{ }_{B} C \rightarrow Z
$$

is surjective. But

$$
\operatorname{dim}\left(\overline{B w B} \times{ }_{B} C\right)=l(w)+\operatorname{dim}(C)=\operatorname{codim}_{X}(Y)+\operatorname{dim}(C)=\operatorname{dim}(Z)
$$

and therefore, $\pi_{C, w}$ is generically finite: $w \in W(C)$. 
Assume now that $Y$ meets properly any $G$-invariant subvariety of $Z$. Let $C \in \mathcal{C}(Y \cap Z)$. We prove that $W(C) \subset W(Y)$ and $G C=Z$ by induction on $\operatorname{codim}_{X}(Y)$. If $\operatorname{codim}_{X}(Y)=0$ then $Y=X$ whence $C=Z$ and $W(C)=W(Y)=$ $\{1\}$. If $\operatorname{codim}_{X}(Y)>0$ then $C \neq Z$. It follows that $G C=Z$ : indeed, because $C \in \mathcal{C}(Y \cap G C)$ and $Y$ meets properly $G C$, we have

$$
\operatorname{codim}_{G C}(C)=\operatorname{codim}_{X}(Y)=\operatorname{codim}_{Z}(C)
$$

which implies that $\operatorname{dim}(G C)=\operatorname{dim}(Z)$. In particular, $G C \neq C$. Let $w \in W(C)$; then $w \neq 1$. Write $w=\tau s_{\alpha}$ where $\alpha \in \Delta$ and $l(w)=l(\tau)+1$. Then $P_{\alpha} C \neq C$, whence $P_{\alpha} Y \neq Y$; otherwise, we would have $P_{\alpha} C \subset Y \cap Z$ and $\operatorname{dim}\left(P_{\alpha} C\right)=$ $\operatorname{dim}(C)+1$ which is impossible because $Y$ meets $Z$ properly. It follows that $P_{\alpha} Y$ meets $Z$ properly and that

$$
\mathcal{C}\left(P_{\alpha} Y \cap Z\right)=\left\{P_{\alpha} C \mid C \in \mathcal{C}(Y \cap Z) \text { and } P_{\alpha} C \neq C\right\} .
$$

Similarly, $P_{\alpha} Y$ meets properly any $G$-invariant subvariety of $Z$. By induction, we have $W\left(P_{\alpha} Y\right) \supset W\left(P_{\alpha} C\right)$. Now the latter contains $\tau$, whence $w \in W(Y)$.

(ii) Let $C \in \mathcal{C}(Y \cap Z)$; then $W(C) \subset W(Y)$ by (i). If moreover $C^{\prime} \in \mathcal{C}(Y \cap$ $Z) \backslash\{C\}$ then $Z=G C=G C^{\prime}$. Because the $G$-action on $Z$ is cancellative, the sets $W(C)$ and $W\left(C^{\prime}\right)$ are disjoint.

Consider now $D \in \mathcal{B}(Z)$ such that $W(D)$ meets $W(Y)$. We prove that $D \in$ $\mathcal{C}(Y \cap Z)$ by induction on $\operatorname{codim}_{Z}(D)$. If $\operatorname{codim}_{Z}(D)=0$ then $D=Z$ whence $1 \in W(Y)$ and $Y=X$. If $\operatorname{codim}_{Z}(D)>0$, choose $w \in W(D) \cap W(Y)$ and write $w=\tau s_{\alpha}$ where $\alpha \in \Delta$ and $l(w)=l(\tau)+1$. Then $P_{\alpha} D \neq D$ and $P_{\alpha} Y \neq Y$. Because $\tau \in W\left(P_{\alpha} D\right) \cap W\left(P_{\alpha} Y\right)$ and $P_{\alpha} Y$ meets properly any $G$-invariant subvariety of $Z$, we have $P_{\alpha} D \in \mathcal{C}\left(P_{\alpha} Y \cap Z\right)$ by the induction assumption. Thus, there exists $C \in \mathcal{C}(Y \cap Z)$ such that $P_{\alpha} D=P_{\alpha} C \neq C$. Because the $G$-action on $Z$ is cancellative, we have $D=C$.

We consider the map

$$
\begin{aligned}
\pi: P_{\alpha} \times_{B} X & \rightarrow X \\
(g, x) B & \mapsto g x,
\end{aligned}
$$

a proper, flat morphism (indeed, $\pi$ identifies with projection $P_{\alpha} / B \times X \rightarrow X$ under the isomorphism $\left.P_{\alpha} \times_{B} X \simeq P_{\alpha} / B \times X\right)$. We have in the Chow group of $X$ :

$$
\pi_{*}\left[P_{\alpha} \times_{B} Y^{\prime}\right]=d\left(Y^{\prime}, s_{\alpha}\right)\left[P_{\alpha} Y^{\prime}\right]
$$

for any $Y^{\prime} \in \mathcal{B}(X)$. Moreover,

$$
\pi^{*}[Z]=\left[P_{\alpha} \times_{B} Z\right]
$$

because $Z$ is $P_{\alpha}$-invariant. It follows that

$$
\begin{aligned}
d\left(Y, s_{\alpha}\right)\left[P_{\alpha} Y\right][Z]=\pi_{*}\left(\left[P_{\alpha} \times{ }_{B} Y\right] \pi^{*}[Z]\right)=\pi_{*}\left(\left[P_{\alpha} \times{ }_{B} Y\right]\left[P_{\alpha} \times_{B} Z\right]\right)= \\
\sum_{C \in \mathcal{C}(Y \cap Z)} i(C, Y \cdot Z) \pi_{*}\left[P_{\alpha} \times_{B} C\right]=\sum_{C \in \mathcal{C}(Y \cap Z)} d\left(C, s_{\alpha}\right) i(C, Y \cdot Z)\left[P_{\alpha} C\right]
\end{aligned}
$$


in the Chow group of $P_{\alpha} Y \cap Z$. Indeed, for $C \in \mathcal{C}(Y \cap Z)$, the varieties $P_{\alpha} \times{ }_{B} Y$ and $P_{\alpha} \times{ }_{B} Z$ intersect properly along $P_{\alpha} \times{ }_{B} C$ with multiplicity $i(C, Y \cdot Z)$. Recall that the top-dimensional Chow group of $P_{\alpha} Y \cap Z$ is freely generated by the classes of the elements of $\mathcal{C}\left(P_{\alpha} Y \cap Z\right)$. Considering the coefficient of $\left[P_{\alpha} C\right]$ in the equalities above, we obtain

$$
d\left(Y, s_{\alpha}\right) i\left(P_{\alpha} C, P_{\alpha} Y \cdot Z\right)=d\left(C, s_{\alpha}\right) i(C, Y \cdot Z) .
$$

It follows that $d(C, w) i(C, Y \cdot Z)=d(Y, w)$ for any $w \in W(C)$ and for any $C \in$ $\mathcal{C}(Y \cap Z)$.

Corollary. Assume that $Z$ is a closed $G$-orbit with isotropy group $Q \supset B^{-}$and that $Y$ meets $Z$ properly. Then the irreducible components of $Y \cap Z$ are the $\overline{B \tau Q} / Q$ where $\tau \in W^{L}$ and $\tau^{-1} \in W(Y)$. Moreover, the intersection multiplicity of $Y$ and $Z$ along $\overline{B \tau Q} / Q$ is $d\left(Y, \tau^{-1}\right)$.

\section{4. $B$-invariant subvarieties in regular $G$-varieties}

We begin by recalling the notion of a regular variety, due to Bifet, De Concini and Procesi (see $[\mathrm{BDP}])$.

Definition. A $G$-variety is regular if it satisfies the following conditions:

(i) $X$ is smooth and contains a dense $G$-orbit $X_{G}^{0}$ whose complement is a union of irreducible smooth divisors with normal crossings (the boundary divisors).

(ii) Any $G$-orbit closure in $X$ is the transversal intersection of the boundary divisors which contain it.

(iii) For any $x \in X$, the normal space $T_{x} X / T_{x}(G x)$ contains a dense orbit of the isotropy group $G_{x}$.

Any complete regular variety $X$ is spherical, that is, $X$ contains a dense $B$-orbit $X_{B}^{0}$. Conversely, any spherical homogeneous space admits a regular completion $X$. Moreover, all closed $G$-orbits in $X$ are isomorphic to $G / Q$ where $Q \supset B^{-}$is opposite to the parabolic subgroup $P \supset B$ consisting of all $g \in G$ which leave invariant $X_{B}^{0}$ (see e.g. [BB] 2.2).

Theorem. Let $X$ be a complete regular $G$-variety and let $Y \subset X$ be a B-invariant subvariety.

(i) For any $w \in W(Y)$, we have $w^{-1} \in W^{L}$ where $L$ is the Levi subgroup of $Q$ which contains $T$.

(ii) For any $G$-invariant subvariety $Z \subset G Y$, the intersection of $Y$ and $Z$ is proper in $G Y$, and we have $G C=Z$ for any irreducible component $C$ of $Y \cap Z$.

(iii) If moreover $Z$ is cancellative, then $Y \cap Z$ is the union of all $C \in \mathcal{B}(Z)$ such that $W(C)$ is contained in $W(Y)$. Moreover, the intersection multiplicity of $Y$ and $Z$ along $C$ is $d(Y, w) d(C, w)^{-1}$ for any $w \in W(C)$. In particular, this multiplicity is a power of 2 . 
Proof. Replacing $X$ by the regular $G$-variety $G Y$, we may assume that $Y$ meets $X_{G}^{0}$.

We first prove (ii). Write $Z=X_{1} \cap \cdots \cap X_{c}$ where $X_{1}, \ldots, X_{c}$ are boundary divisors and $c=\operatorname{codim}_{X}(Z)$. Let $i$ be the greatest index such that the intersection $Y \cap X_{1} \cap \cdots \cap X_{i}$ is proper. If $i \neq c$ then there exists $C \in \mathcal{C}\left(Y \cap X_{1} \cap \cdots \cap X_{i}\right)$ such that $C \subset X_{i+1}$. Observe that $C$ is $B$-invariant and that $\operatorname{dim}(C)=\operatorname{dim}(Y)-i$. Choose $w \in W(C)$. Because $G C \subset X_{1} \cap \cdots \cap X_{i+1}$, we have $l(w)+\operatorname{dim}(C) \leq$ $\operatorname{dim}(X)-i-1$ and therefore:

$$
\operatorname{dim} \overline{B w Y} \leq l(w)+\operatorname{dim}(Y)=l(w)+\operatorname{dim}(C)+i \leq \operatorname{dim}(X)-1 .
$$

So $\overline{B w Y}$ is contained in $X \backslash X_{B}^{0}$. The latter has pure codimension 1, because $X_{B}^{0}$ is affine. Thus, there exists an irreducible $B$-invariant divisor $D \subset X$ containing $\overline{B w Y}$; then $D$ is not $G$-invariant because $G Y=X$. In particular, $D$ contains $\overline{B w C}=G C$ and meets $X_{G}^{0}$. But this is impossible in a regular $G$-variety, see e.g. [BB] Proposition 2.2.1. Thus, $i=c$, that is, $Y$ meets $Z$ properly. We conclude by Lemma 1.3.

Now we prove (i). Let $w \in W(Y)$ and let $Z \subset X$ be a closed $G$-orbit; let $z \in Z$ such that $G_{z}=Q$. By Lemma 1.3, there exists $C \in \mathcal{C}(Y \cap Z)$ such that $w \in W(C)$. Then $C=\overline{B \tau z}$ for some $\tau \in W^{L}$, and $w=\tau^{-1}$.

(iii) follows from (i), (ii) and Lemmas 1.1, 1.3.

We apply this result to a study of the intersection numbers $\int_{X}[Y]\left[Y^{\prime}\right]$ where $Y, Y^{\prime}$ are $B$-invariant subvarieties of $X$ of complementary dimensions. In the case where $X=G / B$, the abelian group $A_{*}(X)$ is freely generated by the Schubert cycles $\Omega(w):=[\overline{B w B} / B](w \in W)$. Furthermore, $\int_{G / B} \Omega(w) \Omega\left(w^{\prime}\right) \neq 0$ if and only if $w^{\prime}=w_{0} w$. In this case, the intersection of $\overline{B w B} / B$ and $w_{0} \overline{B w^{\prime} B} / B$ consists of the point $w B / B$ with multiplicity one. This can be generalized as follows.

Corollary. Let $X$ be a complete regular $G$-variety and let $Y, Y^{\prime}$ be $B$-invariant subvarieties such that $\operatorname{dim}(Y)+\operatorname{dim}\left(Y^{\prime}\right)=\operatorname{dim}(X)$ and that $G Y=X$. Then $\int_{X}[Y]\left[Y^{\prime}\right] \neq 0$ if and only if $Y$ meets $w_{0} Y^{\prime}$. In this case, $Y \cap w_{0} Y^{\prime}$ is a unique point fixed by $T$.

Proof. We have $\int_{X}[Y]\left[Y^{\prime}\right]=\int_{X}[Y]\left[w_{0} Y^{\prime}\right]$. If this number is non-zero, then $Y$ meets $w_{0} Y^{\prime}$. For the converse, let $\mathcal{O} \subset X$ be a $G$-orbit which meets $Y \cap w_{0} Y^{\prime}$. By Kleiman's transversality theorem (see $[\mathrm{Kl}]$ ), there exists an open dense subset $U$ of $G$ such that for all $g \in U$, the intersection $Y \cap g Y^{\prime} \cap \mathcal{O}$ is non-empty of dimension

$$
\operatorname{dim}(Y \cap \mathcal{O})+\operatorname{dim}\left(Y^{\prime} \cap \mathcal{O}\right)-\operatorname{dim}(\mathcal{O}):=n .
$$

Then $U$ meets $B w_{0} B$. Because $Y$ and $Y^{\prime}$ are $B$-invariant, it follows that $U$ contains $w_{0}$. On the other hand, we have by the theorem above:

$$
\operatorname{dim}(Y \cap \mathcal{O})=\operatorname{dim}(\mathcal{O})-\operatorname{dim}(X)+\operatorname{dim}(Y)
$$


and

$$
\operatorname{dim}\left(Y^{\prime} \cap \mathcal{O}\right)=\operatorname{dim}(\mathcal{O})-\operatorname{dim}\left(G Y^{\prime}\right)+\operatorname{dim}\left(Y^{\prime}\right)
$$

It follows that

$$
n=\operatorname{dim}(\mathcal{O})-\operatorname{dim}\left(G Y^{\prime}\right) .
$$

Because $\mathcal{O} \subset G Y^{\prime}$ and $n \geq 0$, this forces $n=0$ and $\overline{\mathcal{O}}=G Y^{\prime}$. Therefore, $Y \cap w_{0} Y^{\prime}$ is a finite subset of $\mathcal{O}$. Because this set is invariant under $B \cap w_{0} B w_{0}^{-1}=T$, it consists of $T$-fixed points.

Let $Y_{B}^{\prime 0}$ be the open $B$-orbit in $Y^{\prime}$, then $\mathcal{O}=G Y_{B}^{\prime 0}$. Set $Y^{\prime \prime}:=\left(Y^{\prime} \cap \mathcal{O}\right) \backslash Y_{B}^{\prime 0}$. Then $Y^{\prime \prime} \subset \mathcal{O}$ is $B$-invariant and $\operatorname{dim}\left(Y^{\prime \prime}\right)<\operatorname{dim}\left(Y^{\prime}\right)$. By Kleiman's transversality theorem again, $Y \cap w_{0} Y^{\prime \prime}$ is empty. It follows that $Y \cap w_{0} Y^{\prime} \subset w_{0} Y_{B}^{\prime 0}$. But the $B$-orbit $Y_{B}^{\prime 0}$ contains at most one $T$-fixed point. This completes the proof.

Remarks. (i) For $Y$ and $Y^{\prime}$ as above, the intersection $Y \cap w_{0} Y^{\prime}$ may be non transversal. Consider for example $G=\operatorname{PGL}(2)$ acting on the space $V$ of quadratic forms in $x, y$ by linear change of variables. Let $X$ be the projectivization of $V$ and let $Y$ (resp. $Y^{\prime}$ ) be the image in $X$ of forms divisible by $x$ (resp. of degenerate forms). Then $G Y=X$ and $Y$ meets $w_{0} Y^{\prime}$ at the image of $x^{2}$, with multiplicity 2 .

(ii) If $G Y$ is not equal to $X$, then it is contained in some boundary divisor $X^{\prime} \subset X$. Using the projection formula (see $[\mathrm{F}]$ p. 140), it follows that

$$
\int_{X}[Y]\left[Y^{\prime}\right]=\int_{X^{\prime}}[Y]\left(\left[X^{\prime}\right]\left[Y^{\prime}\right]\right)
$$

Thus, to compute inductively the left-hand side, it is enough to express $\left[X^{\prime}\right]\left[Y^{\prime}\right]$ in terms of classes of $B$-invariant subvarieties, for any boundary divisor $X^{\prime}$ and for any $B$-invariant subvariety $Y^{\prime}$. In the case where $X$ is a regular completion of $G$, this will be done in 3.4 below.

\subsection{Orbit closures of spherical subgroups in flag varieties}

Let $H \subset G$ be a spherical subgroup and let $P \supset B$ be a parabolic subgroup of $G$; then $G / P$ contains only finitely many $H$-orbits. We express the classes of $H$-orbit closures in the Chow group $A_{*}(G / P)$ endowed with its basis of Schubert cycles $[\overline{B w P} / P]\left(w \in W^{L}\right)$.

First we associate to each $H$-invariant subvariety $V \subset G / P$ a $P$-invariant subvariety $\hat{V} \subset G / H$, as follows. Denote by $q_{P}: G \rightarrow G / P$ and $q_{H}: G \rightarrow G / H$ the quotient maps, and by $\iota: G \rightarrow G$ the map $g \mapsto g^{-1}$. Set

$$
\hat{V}:=q_{H} \iota q_{P}^{-1}(V) .
$$

Then $\hat{V} \subset G / H$ is a $P$-invariant subvariety (which implies that $W(\hat{V}) \subset W^{L}$ ) and

$$
V=q_{P} \iota q_{H}^{-1}(\hat{V}) .
$$


If moreover $H$ is connected, then any $P$-invariant subvariety of $G / H$ is obtained in this way.

Theorem. Let $H$ be a spherical subgroup of $G$, let $B$ be a Borel subgroup of $G$ such that $B H$ is open in $G$, and let $P \supset B$ be a parabolic subgroup with Levi subgroup $L \supset T$. Finally, let $V \subset G / P$ be an $H$-invariant subvariety.

(i) For any $w \in W^{L}$ such that $l(w)=\operatorname{codim}_{G / P}(V)$, the Schubert variety $\overline{B w P} / P$ meets $V$ in $d(\hat{V}, w)$ points of multiplicity one, and these points are contained in $B w P / P$.

(ii) We have in $A_{*}(G / P)$ :

$$
[V]=\sum_{w \in W(\hat{V})} d(\hat{V}, w)\left[\overline{B w_{0} w P} / P\right] .
$$

In particular, the coefficient of any $H$-invariant subvariety on any Schubert cycle is zero or a power of 2.

Proof. (i) By [Kl], there exists a non-negative integer $d$ and an open dense subset $U \subset G$ such that for all $g \in U$, the intersection $(g V) \cap \overline{B w P} / P$ consists of $d$ points of multiplicity one, contained in $B w P / P$. Because $U$ meets $B H$ and $V$ (resp. $\overline{B w P} / P$ ) is invariant under $H$ (resp. $B$ ), it follows that $V \cap \overline{B w P} / P$ consists of $d$ points of multiplicity one.

To show that $d=d(\hat{V}, w)$, we first reduce to the case where $P=B$, as follows. Let $p: G / B \rightarrow G / P$ be the canonical map. Then $p^{-1}(V)$ is an $H$ invariant subvariety of $G / B$; on the other hand, restriction of $p$ to $B w B / B$ is an isomorphism onto $B w P / P$ because $w \in W^{L}$. Therefore, we have by the projection formula:

$$
d=\int_{G / P}[V] p_{*}[\overline{B w P} / P]=\int_{G / B}\left[p^{-1}(V)\right][\overline{B w B} / B] .
$$

Write $w=\tau s_{\alpha}$ with $\alpha \in \Delta, \tau \in W$ and $l(w)=l(\tau)+1$. Let $q: G / B \rightarrow G / P_{\alpha}$ be the canonical map. Then $q$ is a $\mathbf{P}^{1}$-fibration and

$$
q^{*} q_{*}[\overline{B \tau B} / B]=\left[q^{-1} q(\overline{B \tau B} / B)\right]=[\overline{B w B} / B] .
$$

Moreover, we have $q^{-\widehat{1} q(V)}=P_{\alpha} \hat{V}$. We claim that

$$
q^{*} q_{*}[V]=d\left(\hat{V}, s_{\alpha}\right)\left[q^{-1} q(V)\right] .
$$

Indeed, for $y \in \hat{V}$ generic, $d\left(\hat{V}, s_{\alpha}\right)$ is the number of classes $g B$ such that $g \in P_{\alpha}$ and $g^{-1} y \in \hat{V}$. Therefore, $d\left(\hat{V}, s_{\alpha}\right)$ is the degree of the restriction $\left.q\right|_{V}: V \rightarrow q(V)$. Thus, $q_{*}[V]=d\left(\hat{V}, s_{\alpha}\right)[q(V)]$ which implies our claim.

By the projection formula, we have

$$
\begin{aligned}
d=\int_{G / B}[V] q^{*} q_{*}[\overline{B \tau B} / B] & =\int_{G / P_{\alpha}} q_{*}[V] q_{*}[\overline{B \tau B} / B] \\
= & \int_{G / B}\left(q^{*} q_{*}[V]\right)[\overline{B \tau B} / B]=d\left(\hat{V}, s_{\alpha}\right) \int_{G / B}\left[q^{-1} q(V)\right][\overline{B \tau B}] .
\end{aligned}
$$


By induction over $l(w)$, this implies $d=d(\hat{V}, w)$.

(ii) follows from the fact that the $[\overline{B w P} / P]\left(w \in W^{L}\right)$ are a basis of $A_{*}(G / P)$ and that the dual basis for the intersection pairing

$$
(x, y) \mapsto \int_{G / P} x y
$$

consists of the $\left[\overline{B w_{0} w P} / P\right]\left(w \in W^{L}\right)$.

\subsection{Degenerations of orbit closures to $B$-invariant cycles}

The results of 1.4 and 1.5 are related by the following construction. Let $X$ be a regular completion of the spherical homogeneous space $G / H$. Let $P \supset B$ be a parabolic subgroup and let $V \subset G / P$ be an $H$-invariant subvariety with corresponding $P$-invariant subvariety $\hat{V} \subset G / H$. Denote by $Y \subset X$ the closure of $\hat{V}$. Consider the maps

$$
\begin{aligned}
\pi: G \times{ }_{P} Y & \rightarrow X \\
(g, y) P & \mapsto g y
\end{aligned}
$$

and

$$
\begin{aligned}
p: G \times_{P} Y & \rightarrow G / P \\
(g, y) P & \mapsto g P
\end{aligned}
$$

Because $\pi$ factors as

$$
\begin{aligned}
& G \times{ }_{P} Y \quad \rightarrow \quad(G / P) \times X \quad \rightarrow \quad X \\
& (g, y) P \quad \mapsto \quad(g P, g y) \quad \mapsto g y,
\end{aligned}
$$

the fibers of $\pi$ identify to closed subschemes of $G / P$ via $p_{*}$.

Denote by $x \in X$ the base point of $G / H$. Choose a closed $G$-orbit $Z \subset X$ and denote by $z \in Z$ the fixed point of $B^{-}$. Then, for a suitable choice of $T$, there exists a $T$-invariant affine subvariety $A \subset X$ which is transversal to $Z$ at $z$ (see e.g. [BB] 2.3). It follows that $A$ is $T$-equivariantly isomorphic to a $T$-module with linearly independent weights. So we can choose a smooth curve $\gamma \subset X$ isomorphic to affine line, transversal to $Z$ at $z$ and containing $x$ (for example, the closure in $A$ of a generic one-parameter subgroup of $T$ will do).

Proposition. Notation being as above, $\pi$ is equidimensional, and $\pi^{-1}(\gamma)$ is irreducible. Moreover, we have in $A_{*}(G / P)$ :

$$
p_{*}\left[\pi^{-1}(x)\right]=[V], p_{*}\left[\pi^{-1}(z)\right]=\sum_{w \in W(Y)} d(\hat{V}, w)\left[\overline{B w_{0} w P} / P\right] .
$$

Proof. Observe that

$$
\pi^{-1}(x)=\{(g, y) P \mid y \in Y, g y=x\}=\left\{\left(g, g^{-1} x\right) P \mid g^{-1} x \in Y\right\}
$$


(as sets) so that

$$
p\left(\pi^{-1}(x)\right)=\left\{g P \mid g^{-1} x \in Y\right\}=V .
$$

Because $\pi^{-1}(x)$ is a general fiber, it is reduced; because $\left.p\right|_{\pi^{-1}(x)}: \pi^{-1}(x) \rightarrow V$ is bijective, we have $p_{*}\left[\pi^{-1}(x)\right]=[V]$.

Similarly, we obtain

$$
p\left(\pi^{-1}(z)\right)=\left\{g P \mid g^{-1} z \in Y \cap Z\right\}=\bigcup_{w \in W(Y)} \overline{Q w P} / P=\bigcup_{w \in W(Y)} \overline{B^{-} w P} / P
$$

by using Theorem 1.4 (iii). It follows that $\pi$ is equidimensional.

Set $\dot{\gamma}:=\gamma \backslash\{z\}$ and $\Gamma:=\overline{\pi^{-1}(\dot{\gamma})}$. Then $\Gamma$ is irreducible so that restriction $\pi: \Gamma \rightarrow \gamma$ is flat and that

$$
p_{*}\left[\pi^{-1}(z) \cap \Gamma\right]=p_{*}\left[\pi^{-1}(x)\right]=[V]
$$

in $A_{*}(G / P)$. Therefore,

$$
p_{*}\left[\pi^{-1}(z) \cap \Gamma\right]=\sum_{w \in W(Y)} d(\hat{V}, w)\left[\overline{B^{-} w P} / P\right]
$$

by Theorem 1.5. Because the irreducible components of $p\left(\pi^{-1}(z) \cap \Gamma\right)$ are irreducible components of $p\left(\pi^{-1}(z)\right)$, this forces $p\left(\pi^{-1}(z) \cap \Gamma\right)=p\left(\pi^{-1}(z)\right)$, that is, the set $\pi^{-1}(z)$ is contained in $\Gamma$. Thus, $\pi^{-1}(\gamma)=\Gamma$ is irreducible and

$$
p_{*}\left[\pi^{-1}(z)\right]=\sum_{w \in W(Y)} d(\hat{V}, w)\left[\overline{B^{-} w P} / P\right] .
$$

Question. Is $\pi$ flat ? Because $\pi$ is equidimensional and $X$ is smooth, the answer would be positive if $Y$ were Cohen-Macaulay. Is the latter true?

\section{Orbit closures in regular group completions}

\subsection{Regular group completions}

Consider the connected reductive group $G$ as a homogeneous space under $G \times G$ for the action given by left and right multiplication: $\left(g_{1}, g_{2}\right) \gamma=g_{1} \gamma g_{2}^{-1}$. Then the isotropy group of the identity is the diagonal diag $G$. By the Bruhat decomposition, $G$ is the disjoint union of the $\left(B \times B^{-}\right)$-orbits $B w B^{-}(w \in W)$. In particular, $G$ is spherical with open $\left(B \times B^{-}\right)$-orbit $B B^{-}$.

Let $X$ be a $(G \times G)$-equivariant completion of $G$ which is regular in the sense of 1.4. We describe the $(G \times G)$-invariant subvarieties $Z$ of $X$. By Proposition A1 
below, there is a unique $z \in Z$ such that $z$ is the limit of a one-parameter subgroup of $T$, and that the orbit $\left(B \times B^{-}\right) z$ is open in $Z$; we refer to $z$ as the base point of $Z$. Moreover, there exists a unique parabolic subgroup $P:=P(Z) \supset B$ with opposite parabolic subgroup $Q \supset B^{-}$and Levi subgroup $L:=L(Z)=P \cap Q$ such that the isotropy group $(G \times G)_{z}$ is the semi-direct product of $R_{u}(Q) \times R_{u}(P)$ with $\operatorname{diag} L \times(C \times\{1\})_{z}$ where $C$ denotes the connected center of $L$. Finally,

$$
Z=(G \times G) \times{ }_{(Q \times P)} Z^{\prime}
$$

where $Z^{\prime}=\overline{(L \times L) z}$ is a regular completion of a quotient of $L$ by a central torus. In particular, all closed $(G \times G)$-orbits in $X$ are isomorphic to $G / B^{-} \times G / B$.

Now we describe the $\left(B \times B^{-}\right)$-invariant subvarieties $Y$ in $X$. Let $y$ be the base point of the $(G \times G)$-invariant subvariety $(G \times G) Y$ and let $P(Y)$ be the corresponding parabolic subgroup with Levi subgroup $L(Y) \supset T$. Then, by 1.2, we have

$$
Y=\overline{\left(B \times B^{-}\right)(\sigma, \tau) Y^{\prime}}
$$

for $\sigma, \tau \in W^{L(Y)}$ and a $(B \cap L(Y)) \times\left(B^{-} \cap L(Y)\right)$-invariant subvariety $Y^{\prime}$ in $Z^{\prime}$. Moreover, because $y$ is fixed by $\operatorname{diag} L(Y)$, we have

$$
Y^{\prime}=\overline{(B \cap L(Y)) \times\left(B^{-} \cap L(Y)\right)(\rho, 1) y}
$$

for $\rho \in W_{L(Y)}$. Observing that $B \sigma(B \cap L(Y))=B \sigma$ and that $B^{-} \tau(B \cap L(Y))=$ $B^{-} \tau$ because $\sigma, \tau \in W^{L(Y)}$, we conclude that

$$
Y=\overline{\left(B \times B^{-}\right)(w, \tau) y}
$$

where $w=\sigma \rho \in W$ and $\tau \in W^{L(Y)}$ are uniquely determined. If moreover $Y$ meets $G$, then $y=1, \tau=1$ and $Y=\overline{B w B^{-}}$.

Having these descriptions at hand, we can state the following

Theorem. Let $X$ be a regular completion of $G$, let $Y \subset X$ be $a\left(B \times B^{-}\right)$-invariant subvariety, and let $Z \subset(G \times G) Y$ be $a(G \times G)$-invariant subvariety.

(i) $Y$ meets $Z$ properly in $(G \times G) Y$, and all intersection multiplicities are equal to one.

(ii) If moreover $Y=\overline{\left(B \times B^{-}\right)(w, \tau) y}$ as above and $Z$ has base point $z$ and associated parabolic subgroup $P(Z)$, then

$$
Y \cap Z=\bigcup \overline{\left(B \times B^{-}\right)(w v, \tau v) z}
$$

(decomposition into irreducible components) where the union is over all $v \in W_{L(Y)}$ such that $\tau v \in W^{L(Z)}$ and $l(w)=l(w v)+l(v)$. In particular,

$$
\overline{B w B^{-}} \cap Z=\bigcup \overline{\left(B \times B^{-}\right)(w v, v) z}
$$


union over all $v \in W^{L(Z)}$ such that $l(w)=l(w v)+l(v)$.

Proof. We apply the results of 1.2 and 1.4 to the group $G \times G$ with Borel subgroup $B \times B^{-}$, maximal torus $T \times T$ and Weyl group $W \times W$. Recall that $Z$ is induced from a regular completion of a central quotient of a Levi subgroup. Using 1.2 and induction over the semisimple rank of $G$, it follows that the $(G \times G)$-action on $Z$ is cancellative.

In the case where $Y=\overline{\left(B \times B^{-}\right)(w, \tau) y}$, set $L:=L(Y)$ and decompose $w$ as

$$
w=\sigma \rho \in W^{L} W_{L}
$$

Moreover, set

$$
Y^{\prime}:=\overline{(B \cap L) \times\left(B^{-} \cap L\right)(\rho, 1) y} .
$$

Let $\left(w_{1}, w_{2}\right) \in W \times W$ such that $l\left(w_{1}\right)+l\left(w_{2}\right)=\operatorname{codim}_{(G \times G) Y}(Y)=l(w)+l(\tau)$. Then we have by 1.2 :

$$
\begin{aligned}
& \left(w_{1}, w_{2}\right) \in(W \times W)(Y) \Leftrightarrow\left(w_{1} \sigma, w_{2} \tau\right) \in\left(W_{L} \times W_{L}\right)\left(Y^{\prime}\right) \\
& \quad \Leftrightarrow w_{2} \tau \in W_{L} \text { and } \rho=\left(w_{1} \sigma\right)^{-1} w_{2} \tau \Leftrightarrow w_{2} \tau \in W_{L} \text { and } w=w_{1}^{-1} w_{2} \tau .
\end{aligned}
$$

Moreover, $d\left(Y,\left(w_{1}, w_{2}\right)\right)=1$ for all such $\left(w_{1}, w_{2}\right)$. Therefore, by Theorem 1.4, $Y$ meets $Z$ properly in $(G \times G) Y$ with all multiplicities equal to one. Moreover, a $\left(B \times B^{-}\right)$-subvariety $C \subset Z$ is an irreducible component of $Y \cap Z$ if and only if $(W \times W)(C)$ meets $(W \times W)(Y)$; then $(W \times W)(C)$ is contained in $(W \times W)(Y)$. We can write $C=\overline{\left(B \times B^{-}\right)\left(u_{1}, u_{2}\right) z}$ where $u_{1} \in W$ and $u_{2} \in W^{L(Z)}$. By 1.2 again, $(W \times W)(C)$ contains $\left(u_{1}^{-1}, u_{2}^{-1}\right)$. It follows that $u_{2}^{-1} \tau \in W_{L}, w \tau^{-1}=u_{1} u_{2}^{-1}$ and $l\left(u_{1}\right)+l\left(u_{2}\right)=l(w)+l(\tau)$. Thus, we have $\left(u_{1}, u_{2}\right)=(w v, \tau v)$ where $v \in W_{L}$ and $l(w v)+l(\tau v)=l(w)+l(\tau)$. Because $\tau \in W^{L}$, we have $l(\tau v)=l(\tau)+l(v)$ and therefore $l(w v)+l(v)=l(w)$. The converse is obtained by reversing the previous arguments.

Corollary. Notation being as above, any $\left(B \times B^{-}\right)$-invariant subvariety of $X$ is smooth in codimension one. Moreover, $\overline{\left(B \times B^{-}\right)(w, \tau) y}$ is smooth at all points of $\left(B \times B^{-}\right)(w v, \tau v) z$.

Proof. Let $Y \in \mathcal{B}(X)$ and let $Z \subset X$ be a boundary divisor of $(G \times G) Y$. Because $Y$ meets $Z$ properly with multiplicity one, the non-singular locus of $Y$ meets all components of $Y \cap Z$ by [F] 7.2. Therefore, it is enough to show that $Y \cap(G \times G) y$ is non-singular in codimension one, where $y$ is the base point of $(G \times G) Y$.

We use the notation of the proof of the theorem, and we set for simplicity $P(Y):=P$ and $L(Y):=L$. Then the map

$$
\begin{array}{ccc}
\overline{B \sigma Q} \times \overline{B^{-} \tau P} \times Y^{\prime} & \rightarrow & Y \\
\left(g_{1}, g_{2}, x\right) & \mapsto & \left(g_{1}, g_{2}\right) x
\end{array}
$$


is surjective. It follows that any irreducible $\left(B \times B^{-}\right)$-invariant divisor in $Y \cap(G \times$ $G) y$ can be written as $\overline{\left(B \times B^{-}\right) y^{\prime}}$ where

(i) $y^{\prime}=\left(\sigma \rho^{\prime}, \tau\right) y$ with $\rho^{\prime} \in W_{L}$ such that $(B \cap L) \times\left(B^{-} \cap L\right)\left(\rho^{\prime}, 1\right) y$ is a divisor in $Y^{\prime} \cap(L \times L) y$, or

(ii) $y^{\prime}=\left(\sigma^{\prime} \rho, \tau\right) y$ with $\sigma^{\prime} \in W^{L}$ such that $\overline{B \sigma^{\prime} Q}$ is a divisor in $\overline{B \sigma Q}$, or

(iii) $y^{\prime}=\left(\sigma \rho, \tau^{\prime}\right) y$ with $\tau^{\prime} \in W^{L}$ such that $\overline{B^{-} \tau^{\prime} P}$ is a divisor in $\overline{B^{-} \tau P}$.

In case (i), the point $\left(\rho^{\prime}, 1\right) y$ is non-singular in $Y^{\prime}$ by normality of Schubert varieties in $L$, see e.g. [MS]. Moreover, the map

$$
\begin{array}{ccc}
\left(B \sigma \cap \sigma R_{u}(P)\right) \times\left(B^{-} \tau \cap \tau R_{u}(Q)\right) \times Y^{\prime} & \rightarrow & Y \\
\left(g_{1}, g_{2}, x\right) & \mapsto \quad\left(g_{1}, g_{2}\right) x
\end{array}
$$

is an open immersion, and its image contains $y^{\prime}=(\sigma, \tau)\left(\rho^{\prime}, 1\right) y$. This implies our claim.

In case (ii), the point $\sigma^{\prime}$ is non-singular in $\overline{B \sigma Q}$ by normality of Schubert varieties in $G$. Therefore, the set

$$
G\left(\sigma, \sigma^{\prime}\right):=\left\{g \in R_{u}(P) \mid \sigma^{\prime} g \in B \sigma Q \cup B \sigma^{\prime} Q\right\}
$$

is a locally closed, smooth subvariety of $G$ containing 1 . Moreover, the map

$$
\begin{array}{ccc}
G\left(\sigma, \sigma^{\prime}\right) & \rightarrow \overline{B \sigma Q / Q} \\
g & \mapsto \sigma^{\prime} g Q / Q
\end{array}
$$

is an open immersion. It follows that the induced map

$$
G\left(\sigma, \sigma^{\prime}\right) \times\left(B^{-} \tau \cap \tau R_{u}(Q)\right) \times(B \cap L) \times\left(B^{-} \cap L\right)(\rho, 1) y \rightarrow Y^{\prime}
$$

is an open immersion as well, which implies our claim.

Finally, case (iii) is similar to case (ii).

The second assertion follows from the fact that $\left(B \times B^{-}\right)(w v, \tau v) z$ is an open orbit of $B \times B^{-}$in $\overline{\left(B \times B^{-}\right)(w, \tau) y} \cap Z$ and from the criterion for multiplicity one (see $[\mathrm{F}] 7.2$ ).

Question. Is it true that all $\left(B \times B^{-}\right)$-invariant subvarieties of regular group completions are normal ? By the Corollary above, this would hold if they were Cohen-Macaulay.

\subsection{Tangent spaces to closures of double classes}

The group PGL(2) has a unique regular completion $X$ : the projectivization of the space of $2 \times 2$ matrices where $\mathrm{GL}(2) \times \mathrm{GL}(2)$ acts by left and right multiplication. Moreover, the closure in $X$ of the standard Borel subgroup $B \subset \operatorname{PGL}(2)$ is the 
projectivization of the subspace of upper triangular matrices. So $\bar{B}$ is non-singular; but this case is exceptional, as we will see. To state our result, we need the following

Definition. A simple root $\alpha$ is isolated (in the Dynkin diagram of $G$ ) if $\alpha$ is orthogonal to all other simple roots.

Observe that $G$ has no isolated simple root if and only if the adjoint group of $G$ does not contain PGL(2) as a direct factor.

Theorem. Let $X$ be a regular completion of $G$, let $w \in W$ and let $x \in X$ be a fixed point of $B \times B^{-}$.

(i) If $w(\alpha) \in \Phi^{+}$whenever $\alpha$ is an isolated simple root, then the tangent space of $\overline{B w B^{-}}$at $x$ is equal to the tangent space of $X$ at $x$.

(ii) If $w$ is not a product of reflections associated to isolated simple roots, then $\overline{B w B^{-}}$is singular at $x$.

Proof. (i) Set $Z:=(G \times G) x$. Observe that the tangent space $T_{x} \overline{B w B^{-}}$contains $T_{x}\left(\overline{B w B^{-}} \cap Z\right)$ and that $x=\left(w_{0}, w_{0}\right) z$ where $z$ is the base point of $Z$. Applying Theorem 2.1, we obtain

$$
\overline{B w B^{-}} \cap Z \supset \overline{\left(B \times B^{-}\right)(w, 1) z} \cup \overline{\left(B \times B^{-}\right)\left(1, w^{-1}\right) z} \supset(1 \times G) x \cup(G \times 1) x .
$$

It follows that $T_{x} \overline{B w B^{-}}$contains $T_{x} Z$.

Now we show that the quotient space $T_{x} \overline{B w B^{-}} / T_{x} Z$ is equal to the normal space $T_{x} X / T_{x} Z$. By Proposition A1 below, the point $x$ has an open affine $(T \times T)$ invariant neighborhood $X_{x}$ in $X$, which is $(T \times T)$-equivariantly isomorphic to the space of a representation of $T \times T$. Let $X_{1}, \ldots, X_{r}$ be the boundary divisors of $X$ which contain $Z$. Then, for $1 \leq i \leq r$, the divisor $X_{i} \cap X_{x}$ has an equation $f_{i} \in \mathbf{C}\left[X_{x}\right]$ (the algebra of regular functions over $X_{x}$ ) which is unique up to scalar multiplication. In particular, each $f_{i}$ is an eigenvector of $T \times T$; let $\chi_{i}$ be the opposite of its weight. Because $X$ is regular, the characters $\chi_{1}, \ldots, \chi_{r}$ are linearly independent, and

$$
T_{x} X=T_{x} Z \oplus \bigoplus_{i=1}^{r} L_{i}
$$

where each $L_{i}$ is a $(T \times T)$-invariant line with weight $\chi_{i}$. Moreover, the weights of $T \times T$ in $T_{x} Z$ are the $(-\beta, 0)$ and $(0, \beta)$ for $\beta \in \Phi^{+}$.

Let $\mathcal{M}_{X, x}$ be the ideal of $x$ in $\mathbf{C}\left[X_{x}\right]$. Because $T_{x} X$ is the dual of $\mathcal{M}_{X, x} / \mathcal{M}_{X, x}^{2}$, we can choose $(T \times T)$-eigenvectors $f_{\beta, 0}, f_{0,-\beta}$ in $\mathcal{M}_{X, x}$ (for $\beta \in \Phi^{+}$) which lift a basis of the dual of $T_{x} Z$. By the graded Nakayama lemma, the ideal $\mathcal{M}_{X, x}$ is generated by the $f_{\beta, 0}, f_{0,-\beta}\left(\beta \in \Phi^{+}\right)$and by $f_{1}, \ldots, f_{r}$.

For $1 \leq i \leq r$, we denote by $\operatorname{res}\left(f_{i}\right)$ the restriction of $f_{i}$ to $\overline{B w B^{-}} \cap X_{x}$. Because $\overline{B w B^{-}}$meets all orbits of $G \times G$ in $X$, each $\operatorname{res}\left(f_{i}\right)$ is a non-zero element of $\mathcal{M}_{\overline{B w B^{-}}, x}$ (the ideal of $x$ in $\mathbf{C}\left[\overline{B w B^{-}} \cap X_{x}\right]$ ). Using the linear independence 
of $\chi_{1}, \ldots, \chi_{r}$, it is enough to show that no $\operatorname{res}\left(f_{i}\right)$ is contained in $\mathcal{M} \frac{2}{B w B^{-}, x}$. Otherwise, we can write

$$
\chi_{i}=\sum_{j=1}^{r} n_{j} \chi_{j}+(-\beta, \gamma)
$$

where the $n_{j}$ are non-negative integers and where $\beta, \gamma$ are sums of positive roots. By Proposition A2 below, the span of $\chi_{1}, \ldots, \chi_{r}$ intersects the span of $\Phi \times \Phi$ along the span of the $(-\alpha, \alpha)(\alpha \in \Delta)$. It follows that $\gamma=\beta$. By Proposition A2 again, $(-\beta, \beta)$ is in the convex cone generated by $\chi_{1}, \ldots, \chi_{r}$. Writing $(-\beta, \beta)=$ $\sum_{j=1}^{r} m_{j} \chi_{j}$ with non-negative $m_{1}, \ldots, m_{r}$, we obtain

$$
\chi_{i}=\sum_{j=1}^{r}\left(n_{j}+m_{j}\right) \chi_{j} .
$$

By linear independence of $\chi_{1}, \ldots, \chi_{r}$, it follows that $n_{j}=m_{j}=0$ for $j \neq i$ and that $\chi_{i}=(-\beta, \beta)$. By Proposition A2, we must have $\beta=\alpha \in \Delta$. In other words, we have

$$
\chi_{i}=(-\alpha, 0)+(0, \alpha)
$$

for some $\alpha \in \Delta$. Then this decomposition is unique; therefore, we have up to a multiplicative constant:

$$
\operatorname{res}\left(f_{i}\right)=\operatorname{res}\left(f_{\alpha, 0}\right) \operatorname{res}\left(f_{0,-\alpha}\right) .
$$

But $\left(\operatorname{res}\left(f_{i}\right)=0\right)=X_{i} \cap X_{x} \cap \overline{B w B^{-}}$where $X_{i}$ is $(G \times G)$-invariant. It follows that the divisor $\left(\operatorname{res}\left(f_{\alpha, 0}\right)=0\right)$ is $\left(B \times B^{-}\right)$-invariant. Therefore, the same holds for $\left(\operatorname{res}\left(f_{\alpha, 0}\right)=0\right) \cap Z$ and in particular for $\left(\operatorname{res}\left(f_{\alpha, 0}\right)=0\right) \cap(G \times 1) x$.

We claim that $\alpha$ is isolated. To check this, choose root vectors $x_{\beta}(\beta \in \Phi)$ in the Lie algebra of $G$. Denote by $\mathcal{T}$ the tangent space to $\left(\operatorname{res}\left(f_{\alpha, 0}\right)=0\right) \cap(G \times 1) x$ at $x$. Then a basis of $\mathcal{T}$ consists in the $\left(x_{-\beta}, 0\right) x$ where $\beta \in \Phi^{+}$and $\beta \neq \alpha$; by the previous discussion, $\mathcal{T}$ is invariant under the Lie algebra of $B$. If $\alpha$ is not isolated, then there exists $\alpha^{\prime} \in \Delta$ such that $\alpha+\alpha^{\prime}$ is a root. Then $\left[x_{\alpha^{\prime}}, x_{-\alpha-\alpha^{\prime}}\right]$ is a non-zero multiple of $x_{-\alpha}$. Therefore,

$$
x_{\alpha^{\prime}}\left(x_{-\alpha-\alpha^{\prime}}, 0\right) x=\left(\left[x_{\alpha^{\prime}}, x_{-\alpha-\alpha^{\prime}}\right], 0\right) x
$$

is a non-zero multiple of $\left(x_{-\alpha}, 0\right) x$. But $\left(x_{-\alpha-\alpha^{\prime}}, 0\right) x \in \mathcal{T}$ and $\left(x_{-\alpha}, 0\right) x \notin \mathcal{T}$, a contradiction.

Finally, we claim that $w(\alpha) \notin \Phi^{+}$. Let $Z_{\alpha} \subset X$ be the $(G \times G)$-invariant subvariety such that $Z_{\alpha}$ contains $Z$ as a divisor and that the normal space to $Z$ in $Z_{\alpha}$ at $x$ has weight $\chi_{i}=(-\alpha, \alpha)$ (in other words, $Z_{\alpha}=\cap_{j \neq i} X_{j}$ ). Then $P\left(Z_{\alpha}\right)=P_{\alpha}$. Let $z_{\alpha}$ be the base point of $Z_{\alpha}$. By Theorem 2.1, we have

$$
\overline{B w B^{-}} \supset \overline{\left(B \times B^{-}\right)(w, 1) z_{\alpha}} .
$$


If $w(\alpha) \in \Phi^{+}$, then $w^{-1}(\alpha) \in \Phi^{+}$(because $\alpha$ is isolated) and therefore

$$
\overline{\left(B \times B^{-}\right)(w, 1) z_{\alpha}} \supset(w, 1) \overline{\left(B \cap L_{\alpha}\right) \times\left(B^{-} \cap L_{\alpha}\right) z_{\alpha}}=(w, 1) \overline{\left(L_{\alpha} \times L_{\alpha}\right) z_{\alpha}}
$$

where $L_{\alpha}$ is the Levi subgroup of $P_{\alpha}$ which contains $T$. It follows that the tangent space to $\overline{B w B^{-}}$in $X$ at any point of $Z$ contains the normal direction to $Z$ in $Z_{\alpha}$, which contradicts the assumption that $\operatorname{res}\left(f_{i}\right) \in \mathcal{M} \frac{2}{B w B^{-}, x}$.

(ii) Let $I$ be the set of isolated simple roots $\alpha$ such that $w(\alpha) \notin \Phi^{+}$. Then we have by the proof of (i):

$$
\operatorname{dim} T_{x} \overline{B w B^{-}} \geq \operatorname{dim}(Z)+r-|I|=2\left|\Phi^{+}\right|+r-|I| .
$$

On the other hand, we can write

$$
w=\left(\prod_{\alpha \in I} s_{\alpha}\right) w^{\prime}
$$

where $w^{\prime}$ is a product of simple reflections associated to non-isolated simple roots. Then $l(w)=|I|+l\left(w^{\prime}\right)$ and therefore:

$$
\operatorname{dim} \overline{B w B^{-}}=\operatorname{dim}(G)-l(w)=2\left|\Phi^{+}\right|+r-|I|-l\left(w^{\prime}\right) .
$$

Thus, if $\overline{B w B^{-}}$is smooth at $x$, then $w^{\prime}=1$ and $w=\prod_{\alpha \in I} s_{\alpha}$.

Corollary. Let $Y \subset X$ be a $\left(B \times B^{-}\right)$-invariant subvariety. Write $Y=$ $\overline{\left(B \times B^{-}\right)(\rho \sigma, \tau) y}$ with $\rho, \tau \in W^{L(Y)}$ and $\sigma \in W_{L(Y)}$.

(i) If $\sigma(\alpha) \in \Phi^{+}$whenever $\alpha$ is an isolated simple root in the Dynkin diagram of $L(Y)$, then for any $x \in Y$, the composite map

$$
T_{x} Y \rightarrow T_{x}(G \times G) Y \rightarrow T_{x}(G \times G) Y / T_{x}(G \times G) x
$$

is surjective.

(ii) If $\sigma$ is not a product of reflections associated to isolated simple roots in the Dynkin diagram of $L(Y)$, then $Y$ is singular in codimension two.

Proof. Because $(G \times G) Y$ is induced from a regular completion of a quotient of $L(Y)$ by a central torus, we easily reduce to the case where $(G \times G) Y=X$. Then $Y=\overline{B w B^{-}}$for some $w \in W$.

(i) For $1 \leq i \leq r$, let $\mathcal{I}_{X_{i}}$ be the ideal sheaf of $X_{i}$ in $X$. Then the set

$$
E_{i}:=\left\{x \in Y \mid \mathcal{I}_{X_{i}, x} \subset \mathcal{I}_{Y, x}+\mathcal{M}_{X, x}^{2}\right\}
$$

is closed and $\left(B \times B^{-}\right)$-invariant. By the theorem above, $E_{i}$ does not contain any fixed point of $B \times B^{-}$. Therefore, $E_{i}$ is empty: the image of $\mathcal{I}_{X_{i}, x}$ in $\mathcal{O}_{Y, x}$ is never contained in $\mathcal{M}_{Y, x}^{2}$. So the map

$$
T_{x} Y \rightarrow T_{x} X / T_{x} X_{i}
$$


is surjective whenever $x \in Y \cap X_{i}$.

(ii) We show that there exists a boundary divisor $Z$ of $X$ such that $Y \cap Z$ contains two irreducible components $C_{1}$ and $C_{2}$ which meet along a divisor in $C_{1}$ and $C_{2}$, and that $T_{x} Y$ is not contained in $T_{x} Z$ for some $x \in C_{1} \cap C_{2}$. Then the tangent space $T_{x} Y$ contains $T_{x}\left(C_{1} \cup C_{2}\right)$ and surjects to $T_{x} X / T_{x} Z$. Therefore, we have

$$
\operatorname{dim}\left(T_{x} Y\right) \geq \operatorname{dim} T_{x}\left(C_{1} \cup C_{2}\right)+1>\operatorname{dim}\left(C_{1}\right)+1=\operatorname{dim}(Y)
$$

and $Y$ is singular along $C_{1} \cap C_{2}$.

By assumption, there exists a non-isolated simple root $\alpha$ such that $w(\alpha) \notin \Phi^{+}$. Then we can write $w=\tau s_{\alpha}$ where $l(w)=l(\tau)+1$. Let $P \supset B$ be the maximal parabolic subgroup such that $-\alpha$ is not a root of $P$; then $s_{\alpha} \in W^{L}$. Let $Z \subset X$ be a boundary divisor such that $P(Z)=P$, and let $z$ be the basis point of $Z$. By Theorem 2.1, $Y \cap Z$ contains $\overline{\left(B \times B^{-}\right)(w, 1) z}$ and $\overline{\left(B \times B^{-}\right)\left(\tau, s_{\alpha}\right) z}$ as irreducible components. Moreover, both components contain $\overline{\left(B \times B^{-}\right)\left(w, s_{\alpha}\right) z}$ as a common divisor. Indeed, let $U_{\alpha} \subset B$ be the one-parameter unipotent subgroup associated to $\alpha$. Then $1 \times U_{\alpha}$ fixes $z$, because $U_{\alpha} \subset R_{u}(P)$. Therefore,

$$
\overline{\left(B \times B^{-}\right)(w, 1) z} \supset\left(1 \times \overline{U_{-\alpha} T U_{\alpha}}\right)(w, 1) z
$$

contains $\left(w, s_{\alpha}\right) z$, as $\overline{U_{-\alpha} T U_{\alpha}}$ contains $s_{\alpha}$. Similarly, as $\tau(\alpha) \in R^{+}$, we have

$$
\overline{\left(B \times B^{-}\right)\left(\tau, s_{\alpha}\right) z} \supset \overline{\left(U_{\tau(\alpha)} T \times 1\right)\left(\tau, s_{\alpha}\right) z}=\left(\tau, s_{\alpha}\right) \overline{\left(U_{\alpha} T U_{-\alpha} \times 1\right) z}
$$

and the latter contains $\left(\tau s_{\alpha}, s_{\alpha}\right) z=\left(w, s_{\alpha}\right) z$. Finally, as $\alpha$ is not isolated, $T_{x} Y$ is not contained in $T_{x} Z$, by the proof of the theorem above.

Remark. The results of this section do not extend to regular completions of arbitrary spherical homogeneous spaces. For example, let $G=\mathrm{SO}(n)$ act on $\mathbf{C}^{n}$ by its standard representation and let $X=\mathbf{P}^{n-1}$ be the projectivization of $\mathbf{C}^{n}$. Then $X$ is a regular completion of the spherical homogeneous space $\mathrm{SO}(n) / \mathrm{O}(n-1)$ by a homogeneous divisor $Z$, the quadric in $\mathbf{P}^{n-1}$. Choose a Borel subgroup $B \subset G$ and a $B$-fixed point $x \in Z$. Let $Y \subset \mathbf{P}^{n-1}$ be the tangent hyperplane of $Z$ at $x$. Clearly, $Y$ is non-singular, $B$-invariant and not contained in $Z$; but $T_{x} Y$ is equal to $T_{x} Z$.

\subsection{Closures of parabolic subgroups}

We describe how the closure of a parabolic subgroup meets a closed orbit in a regular completion of $G$. As an application, we construct a degeneration of the diagonal of a flag variety to a sum of Schubert cycles.

Proposition. Let $X$ be a regular completion of $G$, let $P \supset B$ be a parabolic subgroup of $G$ with Levi subgroup $L \supset T$ and let $Z \subset X$ be a closed $(G \times G)$-orbit 
with $\left(B^{-} \times B\right)$-fixed point $z$. Then

$$
\bar{P} \cap Z=\bigcup_{w \in W, w^{-1} \in W^{L}} \overline{(B \times B)\left(w, w_{0, L} w\right) z}=\bigcup_{w \in W, w^{-1} \in W^{L}} \overline{(P \times P)(w, w) z}
$$

(decomposition into irreducible components). If moreover no isolated simple root of $G$ is a root of $L$, then

$$
\bar{P}^{\mathrm{reg}} \cap Z=\bigcup_{w \in W, w^{-1} \in W^{L}}(P \times P)(w, w) z
$$

where $\bar{P}^{\text {reg }}$ denotes the non-singular locus of $\bar{P}$.

Proof. Observe that

$$
\bar{P}=\overline{B w_{0, L} B}=\overline{B w_{0, L} w_{0} B^{-} w_{0}} .
$$

Applying Theorem 2.1, we obtain

$$
\bar{P} \cap Z=\bigcup \overline{(B \times B)\left(u, w_{0} v\right) z}
$$

union over all $(u, v) \in W \times W$ such that $w_{0, L} w_{0}=u v^{-1}$ and that $l\left(w_{0, L} w_{0}\right)=$ $l(u)+l(v)$. This amounts to $w_{0} v=w_{0, L} u$ and $l\left(w_{0, L}\right)+l(u)=l\left(w_{0, L} u\right)$, that is, $u^{-1} \in W^{L}$. This proves the first assertion.

For the second assertion, let $x \in \bar{P}^{\mathrm{reg}} \cap Z$. Then, because $\bar{P}$ is $(P \times P)$-invariant, $(P \times P) x$ is contained in $\bar{P}^{\text {reg }} \cap Z$. Moving $x$ in its $(P \times P)$-orbit, we may assume that $x=(u, v) z$ with $u, v$ in $W$ and $u^{-1}, v^{-1}$ in $W^{L}$. The irreducible components of $\bar{P} \cap Z$ which contain $x$ are exactly the $\overline{(B \times B)\left(w, w_{0, L} w\right) z}$, such that $w^{-1} \in W^{L}$, $w \leq u$ (for the Bruhat order) and $v \leq w_{0, L} w$. By [De] Lemma 3.5, this amounts to: $w^{-1} \in W^{L}$ and $v \leq w \leq u$. If moreover $u \neq v$ then we may take either $w=u$ or $w=v$. In other words, $x$ belongs to at least two irreducible components of $\bar{P} \cap Z$. Using Corollary 2.2 (ii), we then obtain $\operatorname{dim} T_{x}(\bar{P} \cap Z)>\operatorname{dim} \bar{P}$, a contradiction. So $u=v$ and $x=(u, u) z$.

Remark. Identifying $Z$ with $G / B \times G / B$ instead of $G / B^{-} \times G / B$, we obtain

$$
\bar{P} \cap Z=\bigcup_{w \in W, w^{-1} \in W^{L}} \overline{P w w_{0} B} / B \times \overline{P w B} / B .
$$

Using the construction in 1.6, this leads to a geometric interpretation of a wellknown formula for the class of the diagonal in $A_{*}(G / P \times G / P)$ :

$$
[\operatorname{diag} G / P]=\sum_{w \in W^{L}}\left[\overline{B w P} / P \times \overline{B w_{0} w P} / P\right]
$$


(see $[\mathrm{G}]$ for more on the class of the diagonal). Indeed, consider the canonical map

$$
\begin{array}{rlc}
\pi:(G \times G) \times_{(P \times P)} \bar{P} & \rightarrow \bar{G}=X \\
\left(g_{1}, g_{2}, x\right)(P \times P) & \mapsto \quad\left(g_{1}, g_{2}\right) x
\end{array}
$$

Then, as in 1.6, $\pi$ is equidimensional and its fibers identify with closed subschemes of $G / P \times G / P$ via the projection

$$
p:(G \times G) \times_{(P \times P)} \bar{P} \rightarrow G / P \times G / P .
$$

Moreover, the fiber of $\pi$ at the identity of $G$ is the diagonal $\operatorname{diag} G / P$, and the class of the fiber over the $(B \times B)$-fixed point in $Z$ is

$$
\sum_{w \in W^{L}}\left[\overline{B w P} / P \times \overline{B w_{0} w P} / P\right]
$$

Therefore, the fibers of $\pi$ realize a degeneration of the diagonal to the cycle above.

\section{Intersection theory in regular group completions}

\subsection{Equivariant Chow rings of regular group completions}

Let $X$ be a smooth, projective variety with an action of a torus $T$. To describe the Chow ring $A^{*}(X)$, it is useful to introduce the equivariant Chow ring $A_{T}^{*}(X)$ (see [EG]). Indeed, $A_{T}^{*}(X)$ is a graded algebra over the symmetric algebra $S$ of the character group $X^{*}(T)$. Moreover, $A^{*}(X)$ is the quotient of $A_{T}^{*}(X)$ by its homogeneous ideal generated by all characters of $T$ (see [Br] Corollary 2.3.1).

In turn, the equivariant Chow ring $A_{T}^{*}(X)$ can be described via the localization theorem: the inclusion of the fixed point set $\iota: X^{T} \rightarrow X$ induces a $S$-algebra homomorphism $\iota^{*}: A_{T}^{*}(X) \rightarrow A_{T}^{*}\left(X^{T}\right)$ which is injective over $\mathbf{Q}$ and whose image is determined by the fixed point sets of codimension one subtori of $T$ (see $[\mathrm{Br}]$ Theorem 3.3). In the case where $X$ is a regular embedding of $G$, we consider the action of $T \times T$ with corresponding symmetric algebra $S \times S$. Then, by Proposition A1, the set $X^{T \times T}$ is contained in the union $X_{c}$ of the closed $(G \times G)$ orbits in $X$; moreover, all such orbits are isomorphic to $G / B^{-} \times G / B$. Therefore, $A_{T \times T}^{*}(X)$ embeds into $A_{T \times T}^{*}\left(X_{c}\right)$ and the latter is a product of copies of the ring $A_{T \times T}^{*}\left(G / B^{-} \times G / B\right)$ (see [KK1], [KK2] and [Br] $\S 6$ for descriptions of this ring).

To analyse further $X^{T \times T}$ and $X_{c}$, we consider the torus embedding $\bar{T}$ where $T$ acts by left multiplication. Let $\mathcal{F}$ be the associated fan in $X_{*}(T) \otimes \mathbf{R}$ and let $\mathcal{F}(l)$ be the set of maximal cones of $\mathcal{F}$. Because $\bar{T}$ is invariant under $\operatorname{diag} W$, the fan $\mathcal{F}$ is invariant under $W$, too. Using Proposition A2 below, it follows that $\mathcal{F}=W \mathcal{F}_{+}$where $\mathcal{F}_{+}$is the set of cones of $\mathcal{F}$ which are contained in the positive Weyl chamber. Then $\mathcal{F}_{+}$is a subdivision of this chamber. Moreover, 
$\mathcal{F}_{+}(l)$ parametrizes the closed $(G \times G)$-orbits in $X$, by Proposition A1. So $X^{T \times T}$ is parametrized by $\mathcal{F}_{+}(l) \times W \times W$.

For $\sigma \in \mathcal{F}_{+}(l)$, we denote by $Z_{\sigma} \simeq G / B^{-} \times G / B$ the corresponding closed orbit with base point $z_{\sigma}$, and by

$$
i_{\sigma}: A_{T \times T}^{*}(X) \rightarrow A_{T \times T}^{*}\left(Z_{\sigma}\right)=A_{T \times T}^{*}\left(G / B^{-} \times G / B\right)
$$

the restriction map. Moreover, for $f \in A_{T \times T}^{*}\left(Z_{\sigma}\right)$ and $u, v \in W$, we denote by $f_{u, v}$ the restriction of $f$ to the point $(u, v) z_{\sigma}$. Then $f_{u, v}$ is in $S \otimes S$ (the $(T \times T)$ equivariant Chow ring of the point).

Theorem. For any projective regular embedding $X$ of $G$, the map

$$
\prod_{\sigma \in \mathcal{F}_{+}(l)} i_{\sigma}^{*}: A_{T \times T}^{*}(X) \rightarrow \prod_{\sigma \in \mathcal{F}_{+}(l)} A_{T \times T}^{*}\left(G / B^{-} \times G / B\right)
$$

is injective and its image consists in all families $\left(f_{\sigma}\right)\left(\sigma \in \mathcal{F}_{+}(l)\right)$ in $S \otimes S$, such that

(i) $f_{\sigma, u s_{\alpha}, v s_{\alpha}} \equiv f_{\sigma, u, v}\left(\bmod (u(\alpha), v(\alpha))\right.$ whenever $\alpha \in \Delta$ and the cone $\sigma \in \mathcal{F}_{+}(l)$ has a facet orthogonal to $\alpha$, and that

(ii) $f_{\sigma, u, v} \equiv f_{\sigma^{\prime}, u, v}(\bmod \chi)$ whenever $\chi \in X^{*}(T)$ and the cones $\sigma, \sigma^{\prime} \in \mathcal{F}_{+}(l)$ have a common facet orthogonal to $\chi$.

Proof. We begin by describing all $(T \times T)$-invariant irreducible curves in $X$. Let $\gamma$ be such a curve. Then there exists a unique $(G \times G)$-orbit $\mathcal{O}$ in $X$ such that $\gamma \cap \mathcal{O}$ is open in $\gamma$. Let $z$ be the base point of $\mathcal{O}$ and let $P$ be the associated parabolic subgroup. Then $(G \times G)_{z}$ contains a conjugate of the isotropy subgroup of a general point of $\gamma$; thus, the rank of $(G \times G)_{z}$ is at least $2 l-1$. By Proposition A1, it follows that one of the following three cases occurs.

(1) $P=B$ and $z$ is fixed by $T \times\{1\}$.

(2) $P=P_{\alpha}$ for some $\alpha \in \Delta$, and $(T \times\{1\})_{z}=C \times\{1\}$ (recall that $C$ denotes the connected center of $L$ ).

(3) $P=B$ and $(T \times\{1\})_{z}$ has codimension one in $T \times\{1\}$.

In case (1), the orbit $\mathcal{O}$ is closed in $X$. It follows that $\gamma$ is conjugate in $W \times W$ to a curve joigning $z$ to $\left(s_{\alpha}, 1\right) z$ or to $\left(1, s_{\alpha}\right) z$ (see e.g. $[\mathrm{Br}] 6.5$ ).

In case $(2), \overline{(L \times L) z}:=X^{\prime}$ is an equivariant completion of the group $L / C$ and the latter is isomorphic to $(\mathrm{P}) \operatorname{SL}(2)$. Moreover, $\overline{(G \times G) \gamma}=\overline{(G \times G) z}$ is isomorphic to $(G \times G) \times(Q \times P) X^{\prime}$. Thus, $\sigma$ has a facet orthogonal to $\alpha$, and $\gamma$ is conjugate in $W \times W$ to a $(T \times T)$-invariant curve $\gamma^{\prime} \subset X^{\prime}$ which is not contained in the closed $(G \times G)$-orbit $\mathcal{O}_{\sigma} \subset \overline{\mathcal{O}}$ and which contains the base point $z_{\sigma}$ of $\mathcal{O}_{\sigma}$. So $\gamma^{\prime}$ joins $z_{\sigma}$ to $\left(s_{\alpha}, s_{\alpha}\right) z_{\sigma}$.

In case (3), $\overline{(T \times T) z}:=\gamma^{\prime}$ is a projective line joigning the base points of two closed orbits $(G \times G)$-orbits $\mathcal{O}_{\sigma}, \mathcal{O}_{\sigma^{\prime}}$. Thus, the cones $\sigma, \sigma^{\prime} \in \mathcal{F}_{+}(l)$ have a common facet. Moreover, $\gamma$ is conjugate to $\gamma^{\prime}$ in $W \times W$. 
In particular, the set of irreducible $(T \times T)$-invariant curves in $X$ is finite. Thus, we can apply $[\mathrm{Br}]$ Theorem 3.4 to describe the image of

$$
i^{*}: A_{T \times T}^{*}(X) \rightarrow A_{T \times T}^{*}\left(X^{T \times T}\right):
$$

it is defined by linear congruences $f_{x} \equiv f_{y}(\bmod \chi)$ whenever $x, y \in X^{T \times T}$ are connected by an invariant curve where $T \times T$ acts through the character $\chi$. In our case, the congruences associated to curves of type (1) define the image of $\prod_{\sigma \in \mathcal{F}_{+}(l)} i_{\sigma}^{*}$, whereas curves of type (2) and (3) lead to congruences (i) and (ii).

To obtain a simpler description of $A^{*}(X)$, we consider the $(G \times G)$-equivariant Chow ring $A_{G \times G}^{*}(X)$. The latter is isomorphic over the rationals to the ring of $(W \times W)$-invariants in $A_{T \times T}^{*}(X)$ (see $\left.[\mathrm{EG}]\right)$. Moreover, the rational Chow ring $A^{*}(X)_{\mathbf{Q}}$ is isomorphic to the quotient of $A_{G \times G}^{*}(X)_{\mathbf{Q}}$ by its ideal generated by all homogeneous elements of $S_{\mathbf{Q}}^{W} \otimes S_{\mathbf{Q}}^{W}$ (see [Br] Corollary 6.7).

Corollary 1. The ring $A_{G \times G}^{*}(X)_{\mathbf{Q}}$ consists in all families $\left(f_{\sigma}\right)\left(\sigma \in \mathcal{F}_{+}(l)\right)$ of elements of $S_{\mathbf{Q}} \otimes S_{\mathbf{Q}}$ such that:

(1) $\left(s_{\alpha}, s_{\alpha}\right)\left(f_{\sigma}\right) \equiv f_{\sigma}(\bmod (\alpha, \alpha))$ whenever $\sigma \in \mathcal{F}$ has a facet orthogonal to $\alpha \in \Delta$, and

(2) $f_{\sigma} \equiv f_{\sigma^{\prime}}(\bmod \chi)$ whenever $\sigma, \sigma^{\prime} \in \mathcal{F}_{+}(l)$ have a common facet orthogonal to $\chi \in X^{*}(T)$.

Proof. By [Br] 6.6, the $\operatorname{ring} A_{G \times G}^{*}\left(G / B \times G / B^{-}\right)$is isomorphic to $S \otimes S$ via restriction to $z_{\sigma}$. Moreover, restriction of $f \in S \otimes S$ to $(u, v) z_{\sigma}$ is equal to $(u, v) f_{\sigma}$ where $f_{\sigma}$ denotes restriction of $f$ to $z_{\sigma}$. So relations (i) and (ii) of the Theorem reduce to (1) and (2).

In the case where $G$ is a torus, both statements above reduce to the known description of the equivariant Chow ring of a smooth, complete torus embedding, as the ring of continuous, piecewise polynomial functions on the corresponding fan (see e.g. $[\mathrm{Br}] 5.4$ ). Back to arbitrary $G$, we have the following relation between $A_{G \times G}^{*}(X)$ and $A_{T \times T}^{*}(\bar{T})$, due to Littelmann and Procesi for semisimple adjoint groups and equivariant cohomology (see [LP] Theorem 2.3).

Corollary 2. There is an isomorphism of $S_{\mathbf{Q}} \otimes S_{\mathbf{Q}^{-}}$algebras

$$
A_{G \times G}^{*}(X)_{\mathbf{Q}} \simeq\left(S_{\mathbf{Q}} \otimes A_{T}^{*}(\bar{T})\right)_{\mathbf{Q}}^{W} .
$$

Proof. Let $N$ be the normalizer of $T$ in $G$ and let $\bar{N}$ be its closure in $X$. Observe that $\bar{N}$ is the disjoint union of the $(w, 1) \bar{T}$ for $w \in W$. In particular, $\bar{N}$ contains all fixed points of $T \times T$. It follows that restriction

$$
A_{T \times T}^{*}(X) \rightarrow A_{T \times T}^{*}(\bar{N})
$$


is injective. Furthermore, by the proof of the Theorem above, $\bar{N}$ contains all $(T \times T)$-invariant curves which are not in a closed $(G \times G)$-orbit (that is, which contribute to relations (i) and (ii)). Thus, restriction to $\bar{N}$ induces isomorphisms

$$
A_{T \times T}^{*}(X)^{W \times W} \simeq A_{T \times T}^{*}(\bar{N})^{W \times W} \simeq A_{T \times T}^{*}(\bar{T})^{W} \simeq\left(S \otimes A_{T}^{*}(\bar{T})\right)^{W} .
$$

\subsection{Equivariant classes of $\left(B \times B^{-}\right)$-invariant subvarieties}

Let $X$ be a projective regular embedding of $G$. Recall that the $S \otimes S$-module $A_{T \times T}^{*}(X)$ is generated by equivariant classes of $\left(B \times B^{-}\right)$-invariant subvarieties $Y \subset X$ (see $[\mathrm{Br}] 6.1$ ). By the previous section, the description of these classes reduces to calculating their restriction $i_{\sigma}^{*}[Y]$ to any closed $(G \times G)$-orbit $Z=Z_{\sigma}$.

For this, we write $Y=\overline{\left(B \times B^{-}\right)(w, \tau) y}$ as in 2.1 and we denote by $\sigma_{Y} \in \mathcal{F}_{+}$ the cone associated to $(G \times G) Y$. Then we may assume that $\sigma_{Y}$ is contained in $\sigma$; otherwise $Z$ is not contained in $(G \times G) Y$ and therefore $i_{\sigma}^{*}[Y]=0$. We denote by $\sigma_{Y}(1) \subset \sigma(1)$ the sets of edges (or extremal rays) of these cones. Each $e \in \sigma(1)$ determines a character $\chi_{e}$ of $T$ : the unique primitive character which vanishes at all edges of $\sigma_{Z}$ except at $e$ where it takes non-negative values.

We identify $Z$ to $G / B^{-} \times G / B$. For $w, \tau \in W$, we denote by $\Omega(w, \tau)$ the equivariant class of $\overline{B w B^{-}} / B^{-} \times \overline{B^{-} \tau B} / B$ in $A_{T \times T}^{*}\left(G / B^{-} \times G / B\right)$. These "Schubert classes" are a basis of the $S \otimes S$-module $A_{T \times T}^{*}\left(G / B^{-} \times G / B\right)$. Finally, we denote by

$$
c^{T \times T}: X^{*}(T \times T) \rightarrow A_{T \times T}^{*}(G / B \times G / B)
$$

the characteristic homomorphism (see e.g. [Br] 6.5).

Proposition. Notation being as above, we have

$$
i_{\sigma}^{*}[Y]=\left(\prod_{e \in \sigma_{Y}(1)} c^{T \times T}\left(\chi_{e},-\chi_{e}\right)\right) \sum_{v} \Omega(w v, \tau v)
$$

(sum over all $v \in W_{L(Y)}$ such that $\left.l(w)=l(w v)+l(v)\right)$.

Proof. Recall that $(G \times G) Y$ is the transversal intersection of the boundary divisors of $X$ which contain it, and that these divisors are indexed by the set $\sigma_{Y}(1)$; we denote by $X_{e}$ the boundary divisor corresponding to the edge $e$. Thus, by the self-intersection formula, we have in $A_{T \times T}^{*}((G \times G) Y)$ :

$$
i_{X,(G \times G) Y}^{*}[Y]=\left(\prod_{e \in \sigma_{Y}(1)} c_{1}^{T \times T}\left(X_{e}\right)\right)[Y] .
$$

Moreover, we have

$$
\begin{aligned}
i_{\sigma}^{*}[Y]=i_{X, Z}^{*}[Y] & =i_{(G \times G) Y, Z}^{*} i_{X,(G \times G) Y}^{*}[Y] \\
& =\left(\prod_{e \in \sigma_{Y}(1)} c_{1}^{T \times T}\left(X_{e}\right)\right) i_{(G \times G) Y, Z}^{*}[Y]=\left(\prod_{e \in \sigma_{Y}(1)} c_{1}^{T \times T}\left(X_{e}\right)\right)[Y \cap Z]
\end{aligned}
$$


where the latter equation holds because $Y$ meets $Z$ properly in $(G \times G) Y$. Finally,

$$
[Y \cap Z]=\sum_{v} \Omega(w v, \tau v)
$$

by Theorem 2.1, and each $c_{1}^{T \times T}\left(X_{e}\right)$ restricts to $A_{T \times T}^{*}(Z)$ as $c^{T \times T}\left(\chi_{e},-\chi_{e}\right)$ by Proposition A1 below.

Using the equivariant Chevalley formula (see [KK1] or [Br] 6.6), one can obtain an explicit but complicated expansion of $i_{\sigma}^{*}[Y]$ in the basis of Schubert classes. We now describe the image of $i_{\sigma}^{*}$ in terms of the ring $\mathbf{D}$ of operators of divided differences. Recall that $\mathbf{D}$ is the ring of endomorphisms of the abelian group $S$ generated by multiplications by elements of $S$, and by the operators $D_{\alpha}:=$ $\alpha^{-1}\left(1-s_{\alpha}\right)$ for $\alpha \in \Delta$. The left $S$-module $\mathbf{D}$ has a canonical basis $\left(D_{w}\right)(w \in W)$ where $D_{w}$ is composition of the $D_{\alpha}$ associated to a reduced expression of $w$.

For any scheme $X$ with an action of $G$, the ring $\mathbf{D}$ acts naturally on the equivariant Chow group $A_{*}^{T}(X)$, and we have

$$
D_{w}[Y]=d(Y, w)[\overline{B w Y}]
$$

for any $Y \in \mathcal{B}(X)$ (see $[\mathrm{Br}] 6.3$ ). It follows that $\mathbf{D} \otimes \mathbf{D}$ acts on $A_{T \times T}^{*}(X)$ for any regular completion $X$ of $G$. For brevity, the $D_{w} \otimes D_{\tau}$ will be called the operators of divided differences.

Let $Z \subset X$ be a closed $(G \times G)$-orbit. Define a class $\delta_{G} \in A_{T \times T}^{*}(Z)$ by setting

$$
\delta_{G}=\sum_{w \in W} \Omega\left(w_{0} w, w\right)
$$

Identifying $Z$ with $G / B^{-} \times G / B$, we see that $\delta_{G}$ is the equivariant class of the reduced subscheme

$$
\bigcup_{w \in W} \overline{B w_{0} w B^{-}} / B^{-} \times \overline{B^{-} w B} / B
$$

By $2.3, \delta_{G}$ is closely related to the class of the diagonal in $G / B \times G / B$.

More generally, for any parabolic subgroup $P \supset B$, define $\delta_{P} \in A_{T \times T}^{*}(Z)$ by

$$
\delta_{P}=\sum_{w \in W_{L}} \Omega\left(w_{0} w, w_{0} w_{0, L} w\right)
$$

so that $\delta_{P}$ is the equivariant class of the reduced subscheme

$$
\bigcup_{w \in W_{L}} \overline{B^{-} w B^{-}} / B^{-} \times \overline{B w_{0, L} w B} / B \subset Q / B^{-} \times P / B
$$


This interprets $\delta_{P}$ as the class $\delta$ associated to a Levi subgroup of $P$.

Corollary. Notation being as above, the images under $i_{\sigma}^{*}$ of the equivariant classes of $\left(B \times B^{-}\right)$-invariant subvarieties in $X$ are obtained by applying the operators of divided differences to the classes

$$
\left(\prod_{e \in \sigma(1)} c^{T \times T}\left(\chi_{e},-\chi_{e}\right)\right) \delta_{P(\varphi)}
$$

where $\varphi$ is a face of the cone $\sigma$, and where $P(\varphi) \supset B$ is the parabolic subgroup associated to the set of simple roots which are orthogonal to $\varphi$.

Proof. Let $Y$ be a $\left(B \times B^{-}\right)$-invariant subvariety of $X$ and let $P \supset B$ be the corresponding parabolic subgroup. Observe that $P=P\left(\sigma_{Y}\right)$ : indeed, it follows from Proposition A1 that a Levi subgroup of $P$ is the centralizer of a general element of $\sigma_{Y} \cap X_{*}(T)$.

Let $y$ be the base point of $Y$ as in 2.1. Observe that $(G \times G) y$ contains a unique closed $\left(B \times B^{-}\right)$-orbit, that is,

$$
\mathcal{O}:=\left(B \times B^{-}\right)\left(w_{0}, w_{0} w_{0, L}\right) y .
$$

Moreover, it follows from Theorem 2.1 that

$$
i_{\sigma}^{*}[\overline{\mathcal{O}}]=\delta_{P} .
$$

Finally, we have

$$
Y=\overline{\left(B \times B^{-}\right)\left(w w_{0}, \tau w_{0, L} w_{0}\right) \mathcal{O}}
$$

and $\operatorname{dim}(Y)=l\left(w w_{0}\right)+l\left(\tau w_{0, L} w_{0}\right)+\operatorname{dim}(\mathcal{O})$. Thus, we have

$$
[Y]=\left(D_{w w_{0}} \otimes D_{\tau w_{0, L} w_{0}}\right)[\overline{\mathcal{O}}]
$$

in $A_{T \times T}^{*}(X)$. We conclude by recalling that the action of $\mathbf{D} \otimes \mathbf{D}$ commutes with $i_{\sigma}^{*}(\mathrm{see}[\mathrm{Br}] 6.3)$.

\subsection{The case of the canonical completion of an adjoint semisimple group}

In this section, we consider a connected semisimple adjoint group $G$ and its minimal regular completion $X$ constructed by De Concini and Procesi (see [DP1]). As an application of the Bialynicki-Birula decomposition (see [Bi]), we construct a basis of the abelian group $A_{*}(X)$ consisting of classes of certain $\left(B \times B^{-}\right)$-invariant subvarieties; then, by $[\mathrm{Br}]$ Corollary 3.2.1, the $(T \times T)$-equivariant classes of these varieties are a basis of the $S \otimes S$-module $A_{*}^{T \times T}(X)$. 
Recall that $X$ contains a unique closed $(G \times G)$-orbit $Z$, isomorphic to $G / B^{-} \times$ $G / B$, and that the fan $\mathcal{F}$ associated to $\bar{T}$ consists in all Weyl chambers and their faces. In particular, the cone $\sigma$ asociated to $Z$ is the positive Weyl chamber, and the characters $\chi_{e}$ associated to edges of $\sigma$ are the simple roots. So the faces of $\sigma$ are indexed by the subsets of $\Delta$. For such a subset $I$, we denote by $z_{I}$ the base point of the corresponding $(G \times G)$-orbit and by $P(I) \supset B$ the associated parabolic subgroup; then $I$ is the set of simple roots of the Levi subgroup $L(I)$ of $P(I)$. We set $W_{I}:=W_{L(I)}$ and $W^{I}:=W^{L(I)}$.

Theorem. Notation being as above, the abelian group $A_{*}(X)$ is freely generated by the classes

$$
\left[\overline{\left(B \times B^{-}\right)(w, \tau) z_{I}}\right]
$$

where $w, \tau \in W$ and $I=\left\{\alpha \in \Delta \mid \tau(\alpha) \in \Phi^{+}\right\}$(in particular, $\tau \in W^{I}$ ).

Proof. Let $\rho$ be the one-parameter subgroup of $T$ such that $\langle\rho, \check{\alpha}\rangle=1$ for all $\alpha \in \Delta$. Then $w_{0}(\rho)=-\rho$. Define a one-parameter subgroup $\lambda$ of $T \times T$ by

$$
\lambda(t)=\left(\rho(t), \rho\left(t^{-n}\right)\right)
$$

where $n$ is a large integer. Then $\left(w_{0}, w_{0}\right)(\lambda)=-\lambda$.

We check that the fixed point set of $\lambda$ in $X$ is $X^{T \times T}$ and that the closures of the corresponding "cells"

$$
X(x, \lambda):=\left\{p \in X \mid \lim _{t \rightarrow 0} \lambda(t) p=x\right\}
$$

(where $x \in X^{T \times T}$ ) are the $\overline{\left(B \times B^{-}\right)(w, \tau) z_{I}}$ as above. Then our statement will follow from the Bialynicki-Birula decomposition.

Given $p \in X$, we determine $\lim _{t \rightarrow 0} \lambda(t) p$. We can write $p=\left(b, b^{-}\right)(w, \tau) z_{I}$ with obvious notation. Then $\lambda(t) p=\lambda(t)\left(b, b^{-}\right) \lambda\left(t^{-1}\right) \lambda(t)(w, \tau) z_{I}$ and

$$
\lambda(t)\left(b, b^{-}\right) \lambda\left(t^{-1}\right)=\left(\rho(t) b \rho\left(t^{-1}\right), \rho\left(t^{-n}\right) b^{-} \rho\left(t^{n}\right)\right)
$$

converges as $t \rightarrow 0$ to a point of $T \times T$. Therefore, we may assume that $p=(w, \tau) z_{I}$. Now we consider $z_{I}$ as a point of the Grassmanian $\operatorname{Grass}(\mathcal{G} \oplus \mathcal{G})$ of subspaces of the Lie algebra of $G \times G$, see [DP1] or the Appendix below. Choose root vectors $x_{\beta}(\beta \in \Phi)$ in $\mathcal{G}$. Then it follows from Proposition A1 below that the linear space $(w, \tau) z_{I}$ has a basis consisting of: the $\left(x_{-w(\beta)}, 0\right)$ and $\left(0, x_{\tau(\beta)}\right)\left(\beta \in \Phi^{+} \backslash \Phi_{I}\right)$, the $\left(x_{w(\beta)}, x_{\tau(\beta)}\right)\left(\beta \in \Phi_{I}\right)$ and a basis of $\operatorname{diag} \mathcal{T}$ where $\mathcal{T}$ denotes the Lie algebra of $T$. For $\beta \in \Phi_{I}$ and $n$ large enough, observe that the limit of the line generated by

$$
\lambda(t)\left(x_{w(\beta)}, x_{\tau(\beta)}\right)=\left(t^{\langle\rho, w(\beta)\rangle} x_{w(\beta)}, t^{-n\langle\rho, \tau(\beta)\rangle} x_{\tau(\beta)}\right)
$$

is the line $0 \times \mathcal{G}_{\tau(\beta)}$ if $\tau(\beta) \in \Phi^{+}$(that is, if $\beta \in \Phi^{+}$, because $\tau \in W^{I}$ ), and the line $\mathcal{G}_{w(\beta)} \times 0$ otherwise. It follows that the linear space $\lim _{t \rightarrow 0} \lambda(t)(w, \tau) z_{I}$ has a 
basis consisting of: the $\left(x_{-w(\beta)}, 0\right)$ and $\left(0, x_{\tau(\beta)}\right)\left(\beta \in \Phi^{+}\right)$and a basis of $\operatorname{diag} \mathcal{T}$. In other words, we have

$$
\lim _{t \rightarrow 0} \lambda(t) p=(w, \tau) z .
$$

Thus, the cell $X(\lambda,(w, \tau) z)$ consists of the orbits $\left(B \times B^{-}\right)(w, \tau) z_{I}$ such that $\tau \in W^{I}$.

Remark. Notation being as in 3.2, restriction to $G / B^{-} \times G / B$ of $\overline{\left(B \times B^{-}\right)(w, \tau) z_{I}}$ is equal to

$$
\left(D_{w} \otimes D_{\tau}\right) \prod_{\alpha \in \Delta \backslash I} c^{T \times T}(\alpha,-\alpha) \sum_{w \in W_{I}}\left[\overline{B^{-} w B^{-}} / B^{-} \times \overline{B w_{0, I} w B} / B\right] .
$$

\subsection{Intersection numbers of $\left(B \times B^{-}\right)$-invariant subvarieties}

We maintain the notation and assumptions of 3.3; we determine the intersection numbers $\int_{X}[Y]\left[Y^{\prime}\right]$ for all $\left(B \times B^{-}\right)$-invariant subvarieties $Y, Y^{\prime}$ of complementary dimensions in $X$. More generally, fix a subset $\Delta^{\prime} \subset \Delta$ and set $X^{\prime}:=\overline{(G \times G) z_{\Delta^{\prime}}}$. For $Y, Y^{\prime} \subset X^{\prime}$, we compute $\int_{X^{\prime}}[Y]\left[Y^{\prime}\right]$; we begin with the case where $(G \times G) Y$ and $(G \times G) Y^{\prime}$ meet properly in $X^{\prime}$. This condition translates combinatorially as follows.

Theorem. Let $I, I^{\prime}$ be subsets of $\Delta$ such that $I \cup I^{\prime}=\Delta^{\prime}$ and let

$$
Y=\overline{\left(B \times B^{-}\right)(w, \tau) z_{I}}, Y^{\prime}=\overline{\left(B \times B^{-}\right)\left(w^{\prime}, \tau^{\prime}\right) z_{I^{\prime}}}
$$

be $\left(B \times B^{-}\right)$-invariant subvarieties of $X^{\prime}$ of complementary dimensions. Then

$$
\int_{X^{\prime}}[Y]\left[Y^{\prime}\right]=\left\{\begin{array}{l}
1 \quad \text { if } I \cap I^{\prime}=\emptyset \text { and } w^{-1} w_{0} w^{\prime}=\tau^{-1} w_{0} \tau^{\prime} \in W_{I} W_{I^{\prime}} \\
0 \quad \text { otherwise. }
\end{array}\right.
$$

Proof. By assumption, we have $\operatorname{codim}_{X^{\prime}}(Y)+\operatorname{codim}_{X^{\prime}}\left(Y^{\prime}\right)=\operatorname{dim}\left(X^{\prime}\right)$, that is,

$$
l(w)+l(\tau)+\left|\Delta^{\prime} \backslash I\right|+l\left(w^{\prime}\right)+l\left(\tau^{\prime}\right)+\left|\Delta^{\prime} \backslash I^{\prime}\right|=2 l\left(w_{0}\right)+\left|\Delta^{\prime}\right| .
$$

If $\int_{X^{\prime}}[Y]\left[Y^{\prime}\right] \neq 0$, then $Y \cap\left(w_{0}, w_{0}\right) Y^{\prime}$ is not empty and thus it contains $(T \times T)$ fixed points. But all such points are in $Z$, and the $(T \times T)$-fixed points in $Y \cap Z$ are the $\left(w_{1}, w_{2}\right) z$ where $w_{1} \geq w v$ and $w_{2} \geq \tau v$ for some $v \in W_{I}$ such that $l(w v)+$ $l(\tau v)=l(w)+l(\tau)$ (see Theorem 2.1). If moreover $\left(w_{1}, w_{2}\right) z \in\left(w_{0}, w_{0}\right) Y^{\prime} \cap Z$, then

$$
w v \leq w_{1} \leq w_{0} w^{\prime} v^{\prime} \text { and } \tau v \leq w_{2} \leq w_{0} \tau^{\prime} v^{\prime}
$$


for some $v$ as above and $v^{\prime} \in W_{I^{\prime}}$ such that $l\left(w^{\prime} v^{\prime}\right)+l\left(\tau^{\prime} v^{\prime}\right)=l\left(w^{\prime}\right)+l\left(\tau^{\prime}\right)$. So we obtain

$$
\begin{gathered}
\\
l(w)+l(\tau)=l(w v)+l(\tau v) \leq l\left(w_{1}\right)+l\left(w_{2}\right) \\
\leq l\left(w_{0} w^{\prime} v^{\prime}\right)+l\left(w_{0} \tau^{\prime} v^{\prime}\right)=2 l\left(w_{0}\right)-l\left(w^{\prime}\right)-l\left(\tau^{\prime}\right) .
\end{gathered}
$$

Together with $(*)$, this implies $|I|+\left|I^{\prime}\right| \leq\left|\Delta^{\prime}\right|$ and therefore $I \cap I^{\prime}=\emptyset$. Then equality holds in $(* *)$ : this forces $w v=w_{1}=w_{0} w^{\prime} v^{\prime}$ and $\tau v=w_{2}=w_{0} \tau^{\prime} v^{\prime}$. Thus,

$$
w^{-1} w_{0} w^{\prime}=\tau^{-1} w_{0} \tau^{\prime}=v v^{\prime-1} .
$$

But $v \in W_{I}$ and $v^{\prime} \in W_{I^{\prime}}$ where $I$ and $I^{\prime}$ are disjoint. Therefore, $v$ and $v^{\prime}$ are uniquely determined: $Y \cap\left(w_{0}, w_{0}\right) Y^{\prime}$ contains a unique $(T \times T)$-fixed point $(w v, \tau v) z:=x$. It follows that $Y \cap\left(w_{0}, w_{0}\right) Y^{\prime}$ consists of this point. Moreover, by Corollary 2.1, $\overline{\left(B \times B^{-}\right) x}$ is a component of multiplicity one of $Y \cap Z$, and $\overline{\left(B \times B^{-}\right)\left(w_{0}, w_{0}\right) x}$ is a component of multiplicity one of $Y^{\prime} \cap Z$. Finally, $\overline{\left(B \times B^{-}\right) x}$ and $\left(w_{0}, w_{0}\right) \overline{\left(B \times B^{-}\right)\left(w_{0}, w_{0}\right) x}$ meet transversally at $x$ in $Z$. It follows that $\int_{X^{\prime}}[Y]\left[Y^{\prime}\right]=1$.

The assumptions of the theorem are satisfied if $I=\Delta$; then we obtain easily the following

Corollary. For any $w \in W$ and for any $\left(B \times B^{-}\right)$-invariant subvariety $Y \subset X$, we have

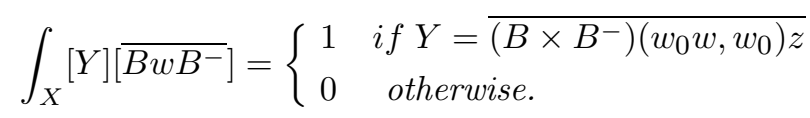

In particular, for the basis of $A_{*}(X)$ constructed in 3.3 , the coordinate function on $\left[\overline{B w B^{-}}\right]$is the scalar product (for the intersection pairing) with $\left[\overline{\left(B \times B^{-}\right)\left(w_{0} w, w_{0}\right) z}\right]$, another element of the basis. But we will see below that the whole basis is not self-dual up to reordering.

Now, to compute $\int_{X}[Y]\left[Y^{\prime}\right]$ for arbitrary $\left(B \times B^{-}\right)$-invariant $Y$ and $Y^{\prime}$, it is enough to determine $\left[X^{\prime}\right][Y]$ for any boundary divisor $X^{\prime} \subset X$ (see the remark in 1.4). This can be done as follows. There exists a unique simple root $\alpha$ such that

$$
X^{\prime}=\overline{(G \times G) z_{\Delta \backslash\{\alpha\}}}:=X^{\alpha} .
$$

Write $Y=\overline{\left(B \times B^{-}\right)(w, \tau) z_{I}}$ as above. If $\alpha \in I$ then $Y$ is not contained in $X^{\alpha}$ and Theorem 2.1 implies that

$$
\left[X^{\alpha}\right][Y]=\sum\left[\overline{\left(B \times B^{-}\right)(w v, \tau v) z_{I \backslash\{\alpha\}}}\right]
$$

(sum over all $v \in W_{I}$ such that $\tau v \in W^{I \backslash\{\alpha\}}$ and that $l(w v)+l(v)=l(w)$ ).

If $\alpha \notin I$ then there exist unique rational numbers $x_{\alpha \beta}(\beta \in \Delta)$ such that

$$
\alpha=\sum_{\beta \in I} x_{\alpha \beta} \beta+\sum_{\gamma \notin I} x_{\alpha \gamma} \omega_{\gamma}
$$


where the $\omega_{\gamma}$ are the fundamental weights of $\Phi$ (that is, $\sum_{\beta \in I} x_{\alpha \beta} \beta$ is the orthogonal projection of $\alpha$ on the linear space generated by $I$ ). Setting

$$
D^{\gamma}:=\overline{B s_{\gamma} B^{-}}
$$

for $\gamma \in \Delta$, we then have in the Picard group of $X$ :

$$
\left[X^{\alpha}\right]=\sum_{\beta \in I} x_{\alpha \beta}\left[X^{\beta}\right]+\sum_{\gamma \notin I} x_{\alpha \gamma}\left[D^{\gamma}\right]
$$

(as can be seen by restricting to $Z$ ). Moreover, each $\left[X^{\beta}\right][Y]$ is determined as above. To compute $\left[D^{\gamma}\right][Y]$, let

$$
p: X^{\alpha} \rightarrow G / Q_{\Delta \backslash\{\alpha\}} \times G / P_{\Delta \backslash\{\alpha\}}
$$

be the projection. Any pair of characters $\lambda, \mu$ of $L_{\Delta \backslash\{\alpha\}}$ defines a homogeneous line bundle on the image of $p$ and we denote by $c(\lambda, \mu)$ its Chern class. Then

$$
D^{\gamma}=p^{*} c\left(\omega_{-\gamma}, \omega_{\gamma}\right)
$$

and we obtain $\left[D^{\gamma}\right][Y]$ as a special case of the following

Lemma. Let $P \supset B$ be a parabolic subgroup of $G$ with Levi subgroup $L \supset T$. Let $X^{\prime}$ be a L-variety, let $X=G \times_{P} X^{\prime}$ be the induced variety with projection $p: X \rightarrow G / P$, and let $Y=\overline{B w Y^{\prime}} \subset X$ where $w \in W^{L}$ and $Y^{\prime} \subset X^{\prime}$ is a $B$-invariant subvariety. Then, for any character $\chi$ of $L$, we have in $A_{*}(X)$ :

$$
p^{*} c(\chi) \cap[Y]=\sum_{\beta}\langle\chi, \check{\beta}\rangle d\left(Y^{\prime}, w s_{\beta}\right)\left[\overline{B w s_{\beta} Y^{\prime}}\right]
$$

(sum over the $\beta \in \Phi^{+}$such that $\left.l\left(w s_{\beta}\right)=l(w)-1\right)$.

Proof. In the equivariant Chow group $A_{*}^{T}(X)$, we have $[Y]=D_{w}\left[Y^{\prime}\right]$. Moreover, $c(\chi)$ lifts to an equivariant Chern class $c^{T}(\chi)$ which commutes with the action of D (see $[\mathrm{Br}] \S 6)$. Thus,

$$
p^{*} c^{T}(\chi) \cap[Y]=D_{w}\left(p^{*} c^{T}(\chi) \cap\left[Y^{\prime}\right]\right) .
$$

Moreover, because $p\left(Y^{\prime}\right)$ is the base point of $G / P$, we have $p^{*} c^{T}(\chi) \cap\left[Y^{\prime}\right]=\chi\left[Y^{\prime}\right]$. Now we conclude by the identity

$$
D_{w}(\chi u)=w(\chi) D_{w}(u)+\sum_{\beta}\langle\chi, \check{\beta}\rangle D_{w s_{\beta}}(u)
$$

for any $u \in A_{*}^{T}(X)$ (see the proof of Proposition 6.6 in $[\mathrm{Br}]$ ). 
Example. Let $G=\operatorname{PGL}(3)$ and let $X$ be the canonical completion of $G$. Let $\alpha, \beta$ be the simple roots; then the boundary divisors of $X$ are $X^{\alpha}$ and $X^{\beta}$ which meet transversally along the closed $(G \times G)$-orbit $Z$. Consider

$$
Y:=\overline{\left(B \times B^{-}\right)\left(s_{\beta}, s_{\alpha}\right) z_{\beta}}, Y^{\prime}:=\overline{\left(B \times B^{-}\right)\left(s_{\beta} s_{\alpha}, s_{\alpha} s_{\beta}\right) z_{\beta}} .
$$

Then $Y$ and $Y^{\prime}$ are contained in $X^{\alpha}$ and their classes in $A_{*}(X)$ belong to the basis constructed in 3.3. We check that

$$
\int_{X}[Y]\left[Y^{\prime}\right]=-1
$$

Recall that $\int_{X}[Y]\left[Y^{\prime}\right]=\int_{X^{\alpha}}\left(\left[X^{\alpha}\right][Y]\right)\left[Y^{\prime}\right]$. Because $\alpha=-\frac{1}{2} \beta+\frac{3}{2} \omega_{\alpha}$, we have in the Picard group of $X$ :

$$
\left[X^{\alpha}\right]=-\frac{1}{2}\left[X^{\beta}\right]+\frac{3}{2}\left[D^{\alpha}\right] .
$$

Moreover,

$$
\left[X^{\beta}\right][Y]=[Y \cap Z]=\left[\overline{\left(B \times B^{-}\right)\left(s_{\beta}, s_{\alpha}\right) z}\right]+\left[\overline{\left(B \times B^{-}\right)\left(1, s_{\beta} s_{\alpha}\right) z}\right] .
$$

By the theorem above, we have

$$
\int_{X^{\alpha}}\left[\overline{\left.\left(B \times B^{-}\right)\left(s_{\beta}, s_{\alpha}\right) z\right]}\left[Y^{\prime}\right]=\int_{X^{\alpha}}\left[\overline{\left(B \times B^{-}\right)\left(1, s_{\beta} s_{\alpha}\right) z}\right]\left[Y^{\prime}\right]=1 .\right.
$$

On the other hand, by the lemma above, $\left[D^{\alpha}\right][Y]$ is a linear combination of classes of $\left(B \times B^{-}\right)$-invariant subvarieties which are not contained in $Z$. Again by the theorem above, we then have $\int_{X^{\alpha}}\left(\left[D^{\alpha}\right][Y]\right)\left[Y^{\prime}\right]=0$ because $Y^{\prime}$ is not contained in $Z$. This implies our assertion.

\section{Appendix: the structure of regular group completions}

We denote by $X_{*}(T)$ the group of one-parameter subgroups of $T$. An element $\lambda \in X_{*}(T)$ is called dominant if the scalar product of $\lambda$ with any positive coroot is non-negative. The group $W$ acts on $X_{*}(T)$ and the set of dominant one-parameter subgroups is a fundamental domain for this action, as it is the intersection of $X_{*}(T)$ with the positive Weyl chamber.

To any $\lambda \in X_{*}(T)$ we associate the subset $G(\lambda) \subset G$ of all $g$ such that $\lambda(t) g \lambda(t)^{-1}$ has a limit in $G$ when $t \rightarrow 0$. Then $G(\lambda)$ is a parabolic subgroup of $G$ with unipotent radical $R_{u} G(\lambda)=\left\{g \in G \mid \lim _{t \rightarrow 0} \lambda(t) g \lambda(t)^{-1}=1\right\}$. Moreover, a Levi subgroup of $G(\lambda)$ is the centralizer $L(\lambda)$ of the image of $\lambda$, and the parabolic subgroups $G(\lambda), G(-\lambda)$ are opposite. Finally, $G(\lambda)$ contains $B$ if and only if $\lambda$ is dominant (see e.g. [MFK] 2.2).

Proposition A1. Let $X$ be a regular completion of $G$ and let $\mathcal{O} \subset X$ be $a(G \times G)$ orbit. 
(i) The closure $\bar{T}$ is smooth and meets $\mathcal{O}$ transversally into a union of $(T \times T)$ orbits permuted transitively by diag $W$.

(ii) There exists a unique $z \in \mathcal{O}$ such that $\left(B \times B^{-}\right) z$ is open in $\mathcal{O}$ and that $z=\lim _{t \rightarrow 0} \lambda(t)$ for some $\lambda \in X_{*}(T)$. The isotropy group $(G \times G)_{z}$ is the semidirect product of $R_{u} G(-\lambda) \times R_{u} G(\lambda)$ with diag $L(\lambda) \times(C(\lambda) \times 1)_{z}$, where $C(\lambda)$ denotes the connected center of $L(\lambda)$. In particular, $G(\lambda)$ depends only on $\mathcal{O}$.

(iii) The $(G \times G)$-equivariant map $\mathcal{O} \rightarrow(G \times G) /(G(-\lambda) \times G(\lambda))$ (defined by inclusion of $(G \times G)_{z}$ into $\left.G(-\lambda) \times G(\lambda)\right)$ extends to the closure $\overline{\mathcal{O}}$ and makes $\overline{\mathcal{O}}$ the induced variety of $\overline{L(\lambda) z}$, a regular completion of the group $L(\lambda) / C(\lambda)_{z}$.

(iv) The orbit $\mathcal{O}$ is closed in $X$ if and only $z$ is fixed by $T \times T$. Then

$$
X_{z}:=\left\{x \in X \mid \lim _{t \rightarrow 0} \lambda(t) x=z\right\}
$$

is an open affine $\left(B \times B^{-}\right)$-invariant subset of $X$. Moreover, $\bar{T}_{z}:=\bar{T} \cap X_{z}$ is isomorphic to affine $l$-space where $T \times T$ acts linearly through $l$ independent weights, and the map

$$
\begin{aligned}
& \varphi: U \times U^{-} \times \bar{T}_{z} \rightarrow X_{z} \\
& \left(g_{1}, g_{2}, x\right) \quad \mapsto \quad\left(g_{1}, g_{2}\right) x
\end{aligned}
$$

is an isomorphism, where $U$ (resp. $U^{-}$) denotes the unipotent radical of $B$ (resp. $\left.B^{-}\right)$.

Proof. Observe that $T$ is the fixed point set of $\operatorname{diag} T$ in $G$. It follows that $\bar{T}$ is a component of the fixed point set $X^{\operatorname{diag} T}$. Therefore, $\bar{T}$ is smooth.

Denote by $k[[t]]$ the ring of formal power series in $t$, and by $k((t))$ its field of fractions. By [MFK] 2.1, any point of $G_{k((t))}$ can be written as $g_{1}(t) \lambda(t) g_{2}(t)$ for some $g_{1}(t), g_{2}(t)$ in $G_{k[[t]]}$ and $\lambda \in X_{*}(T)$. It follows that there exists $\lambda \in X_{*}(T)$ such that $\lim _{t \rightarrow 0} \lambda(t):=z$ exists and belongs to $\mathcal{O}$. Replacing $\lambda$ by $w(\lambda)$ for some $w \in W$, we may assume that $\lambda$ is dominant.

Let $g \in R_{u} G(\lambda)$. Then

$$
\lambda(t)=\left((\lambda(t), 1)(g, g) 1=\left(\lambda(t) g \lambda(t)^{-1}, g\right)(\lambda(t), 1) 1 .\right.
$$

Taking limits at 0 , we obtain $z=(1, g) z$, that is, $1 \times R_{u} G(\lambda)$ fixes $z$. Similarly, $R_{u} G(-\lambda) \times 1$ fixes $z$. Moreover, for $g \in L(\lambda)$, we have $\lambda(t)=(g, g) \lambda(t)$ and therefore $z=(g, g) z$. So $(G \times G)_{z}$ contains $R_{u} G(-\lambda) \times R_{u} G(\lambda)$, diag $L(\lambda)$ and of course $(C(\lambda) \times 1)_{z}$ (which in turn contains $\lambda\left(k^{*}\right) \times 1$ ). Because the product $\left(B \times B^{-}\right)\left(R_{u} G(-\lambda) \times R_{u} G(\lambda)\right) \operatorname{diag} L(\lambda)$ is open in $G \times G$, it follows that $\left(B \times B^{-}\right) z$ is open in $\mathcal{O}=(G \times G) z$.

To show that $(G \times G)_{z}$ is the semidirect product of the groups above, we first consider the case where $\mathcal{O}$ has codimension one in $X$. Then $\operatorname{dim}(G \times G)_{z}=$ $\operatorname{dim}(G)+1$ and therefore the connected component $(G \times G)_{z}^{0}$ is the product of the groups above. It follows that the unipotent radical of $(G \times G)_{z}$ is $R_{u} G(-\lambda) \times$ 
$R_{u} G(\lambda)$. Thus, $(G \times G)_{z}$ is contained in $G(-\lambda) \times G(\lambda)$ (the normalizer in $G \times G$ of its unipotent radical). So $(G \times G)_{z}$ is the product of $R_{u} G(-\lambda) \times R_{u} G(\lambda)$ with $(L(\lambda) \times L(\lambda))_{z}$. Moreover, the latter group contains $(\operatorname{diag} L(\lambda))(C(\lambda) \times 1)_{z}$ as a component, and hence it normalizes the diagonal of the derived subgroup of $L(\lambda)$. It follows that $(L(\lambda) \times L(\lambda))_{z}$ is equal to $\operatorname{diag} L(\lambda) \times(C(\lambda) \times 1)_{z}$.

In the case where the codimension of $\mathcal{O}$ is arbitrary, we replace $X$ by the blow-up $\hat{X}$ of $\overline{\mathcal{O}}$ in $X$; then $\hat{X}$ is a regular completion of $G$. Let $\hat{\mathcal{O}}$ be the open $(G \times G)$-orbit in the exceptional divisor of $\hat{X}$ and let $\hat{z} \in \hat{\mathcal{O}}$ be a point as above. Then $(G \times G)_{\hat{z}}$ is the kernel of the action of $(G \times G)_{z}$ in the normal space at $\mathcal{O}$ at $z$. So $(G \times G)_{\hat{z}}$ is the intersection of kernels of independent characters of $(G \times G)_{z}$. In other words, $(G \times G)_{z}$ is a normal subgroup of $(G \times G)_{\hat{z}}$ and the quotient is a torus; in particular, both groups have the same unipotent radical. Arguing as above, we obtain that $(G \times G)_{z}$ is the product of $R_{u} G(-\lambda) \times R_{u} G(\lambda)$, $\operatorname{diag} L(\lambda)$ and $(C(\lambda) \times 1)_{z}$. This implies that $(T \times T) z$ is a component of $\mathcal{O}^{\operatorname{diag} T}$ and hence of its subset $\bar{T} \cap \mathcal{O}$. Moreover, we have

$$
\begin{gathered}
\operatorname{codim}_{X}(\mathcal{O})=\operatorname{dim}(G)-\operatorname{dim}(G \times G)+\operatorname{dim}(G \times G)_{z}=\operatorname{dim}(C(\lambda) \times 1)_{z} \\
=\operatorname{codim}_{\bar{T}}(T \times T) \cdot z .
\end{gathered}
$$

Therefore, $(T \times T) z$ is a proper component of the intersection of $\bar{T}$ with $\mathcal{O}$. Because $\bar{T}$ is contained in $X^{\operatorname{diag} T}$, we have for tangent spaces:

$$
T_{z} \mathcal{O} \cap T_{z} \bar{T} \subset\left(T_{z} \mathcal{O}\right)^{\operatorname{diag} T}=T_{z}(T \times T) z,
$$

that is, the intersection is transversal at $z$. This proves assertions (i) and (ii).

(iii) is a consequence of (ii) together with the following result.

Lemma. Let $X$ be a regular $G$-variety, let $x \in X_{G}^{0}$, let $P \subset G$ be a parabolic subgroup containing the isotropy group $G_{x}$ and let $p: G \cdot x \rightarrow G / P$ be the map $g \cdot x \mapsto g P$. Then $p$ extends to a $G$-equivariant morphism $X \rightarrow G / P$.

Proof of Lemma. Embed $G / P$ into the projective space $\mathbf{P}(M)$ where $M$ is a simple $G$-module. Let $\delta \subset G / P$ be the intersection of $G / P$ with the $B$-invariant hyperplane in $M$, and let $D \subset X$ be the closure of the pull-back of $\delta$ to $G \cdot x$. Then $D$ contains no $G$-orbit (see e.g. [BB] Proposition 2.2.1). Moreover, the corresponding sheaf $\mathcal{O}_{X}(D)$ is $G$-linearizable, and the $G$-submodule of $\Gamma\left(X, \mathcal{O}_{X}(D)\right)$ generated by the canonical section of $\mathcal{O}_{X}(D)$ identifies to $M^{*}$. Therefore, this space of sections is base-point-free and the corresponding morphism $X \rightarrow \mathbf{P}(M)$ maps $G \cdot x$ to $G / P$, hence $X$ to $G / P$.

Now we prove (iv). If $\mathcal{O}$ is closed, then $(G \times G)_{z}$ is parabolic in $G \times G$, that is, $G(\lambda)=B$ and $z$ is fixed by $T \times T$. Conversely, if $z$ is fixed by $T \times T$, then we must have $C(\lambda)=T$, i.e. $L(\lambda)=T$ and $(G \times G)_{z}=B^{-} \times B$. Thus, $\mathcal{O}$ is closed in $X$. Moreover, $\bar{T}_{z}$ is an affine $(T \times T)$-invariant neighborhood of $z$ in the 
smooth torus embedding $\bar{T}$. Thus, $\bar{T}_{z}$ is isomorphic to affine space of dimension $l$, where $T \times T$ acts linearly through $l$ independent weights. Moreover, $X_{z}$ is $\left(B \times B^{-}\right)$-invariant and contains the identity of $G$. Thus, $X_{z}$ is the open cell of the Bialynicki-Birula decomposition defined by $\lambda$. In particular, $X_{z}$ is isomorphic to affine space. Moreover, the map $\varphi$ restricts to an isomorphism over $U \times U^{-} \times T$, and $\varphi^{-1}(z)$ is a single point. Because $z$ is the unique closed $(T \times T)$-orbit in $X_{z}$, it follows that $\varphi$ is finite. So $\varphi$ is an isomorphism by Zariski's main theorem.

Let $C$ be the center of $G$ and let $G_{a d}:=G / C$ be the corresponding adjoint group. Then $G_{a d}$ has a canonical regular completion $\overline{G_{a d}}$ which can be constructed as follows: for the adjoint action of $G_{a d} \times G_{a d}$ in its Lie algebra $\mathcal{G}_{a d} \oplus \mathcal{G}_{a d}$, the isotropy group of the diagonal $\operatorname{diag} \mathcal{G}_{a d}$ is equal to $G_{a d}$. Then $\overline{G_{a d}}$ is the closure of the $\left(G_{a d} \times G_{a d}\right)$-orbit of $\operatorname{diag} \mathcal{G}_{a d}$ in the corresponding Grassmanian $\operatorname{Grass}\left(\mathcal{G}_{a d} \oplus\right.$ $\left.\mathcal{G}_{a d}\right)$. Moreover, each regular completion $X$ of $G_{a d}$ dominates $\overline{G_{a d}}$, that is, there exists a morphism of $X$ to $\overline{G_{a d}}$ which induces the identity on $G_{a d}$ (then such a morphism is $\left(G_{a d} \times G_{a d}\right)$-equivariant). These results are proved in [DP1] and [St]; they can be slightly generalized as follows.

Proposition A2. For a non-singular $(G \times G)$-equivariant completion $X$ of $G$, the following assertions are equivalent:

(i) $X$ is regular.

(ii) $X$ dominates $\overline{G_{a d}}$.

If (i) holds, let $z \in X$ be a fixed point of $B^{-} \times B$ and let $\chi_{1}, \ldots, \chi_{r}$ be the weights of $T \times T$ in the normal space to $(G \times G) z$ at $z$. Then the convex cone generated by $\chi_{1}, \ldots, \chi_{r}$ contains the $(\alpha,-\alpha)(\alpha \in \Delta)$. Moreover, the intersection of the span of $\chi_{1}, \ldots, \chi_{r}$ with the span of $\Phi \times \Phi$ is the span of the $(\alpha,-\alpha)(\alpha \in \Delta)$.

Proof. (i) $\Rightarrow$ (ii) For any $x \in X$, denote by $(G \times G)_{(x)}$ the kernel of the action of the isotropy group $(G \times G)_{x}$ in the normal space to the orbit $(G \times G) x$ at $x$. Because $X$ is regular, the dimension of $(G \times G)_{(x)}$ is independent of $x$. We claim that

$$
(G \times G)_{(x)} \cap\left(C^{0} \times C^{0}\right)=\operatorname{diag} C^{0}
$$

(where $C^{0}$ denotes the connected center of $C$ ). To check this, it is enough to consider the case where $x=z$ is the base point of its $(G \times G)$-orbit. Then, by Proposition A1, the normal space $T_{z} X / T_{z}(G \times G) z$ identifies to $T_{z} \bar{T} / T_{z}(T \times T) z$, and we have

$$
(G \times G)_{(z)} \cap\left(C^{0} \times C^{0}\right) \subset(T \times T)_{(z)} .
$$

Moreover, it is easy to see that $(T \times T)_{(z)}=\operatorname{diag} T$ which proves our claim.

From this, it follows that the dimension of $(\mathcal{G} \oplus \mathcal{G})_{(x)}+(\mathcal{C} \oplus \mathcal{C})$ is independent of $x$. Therefore, identifying $\operatorname{Grass}\left(\mathcal{G}_{a d} \oplus \mathcal{G}_{a d}\right)$ with the Grassmanian of subspaces of $\mathcal{G} \oplus \mathcal{G}$ which contain $\mathcal{C} \oplus \mathcal{C}$, we obtain a $(G \times G)$-equivariant map

$$
\begin{aligned}
\pi: \quad X & \rightarrow \operatorname{Grass}\left(\mathcal{G}_{a d} \oplus \mathcal{G}_{a d}\right) \\
x & \mapsto(\mathcal{G} \oplus \mathcal{G})_{(x)}+(\mathcal{C} \oplus \mathcal{C}) .
\end{aligned}
$$


Moreover, $\pi(1)$ is identified with the diagonal in $\mathcal{G}_{a d} \oplus \mathcal{G}_{a d}$. Using Proposition A1, it is easy to see that $\pi$ is a morphism. Thus, $\pi$ maps $X$ onto $\overline{G_{a d}}$.

(ii) $\Rightarrow$ (i) By assumption, we have an equivariant morphism $\pi: X \rightarrow \overline{G_{a d}}$. Let $Z \subset X$ be a closed $(G \times G)$-orbit. Then $\pi(Z)$ is the closed $(G \times G)$-orbit in $\overline{G_{a d}}$ which implies that $Z$ is isomorphic to $G / B^{-} \times G / B$. Let $z$ be the $\left(B^{-} \times B\right)$-fixed point in $Z$. Then, as in the beginning of the proof of Proposition A1, we obtain that $z$ is contained in $\bar{T}$ and that $\bar{T}$ is smooth. Moreover, the canonical map $\varphi: U \times U^{-} \times \bar{T}_{z}$ is injective, because the induced map $U \times U^{-} \times\left(\bar{T}_{a d}\right)_{\pi(z)} \rightarrow \overline{G_{a d}}$ is injective. It follows that $\varphi$ is an open immersion; thus, $\bar{T}$ is transversal to $Z$ at $z$. This implies at once that $X$ is regular.

Denote by $A$ (resp. $A_{a d}$ ) the algebra of regular functions over the affine space $\bar{T}_{z}$ (resp. $\left.\left.\overline{\left(T_{a d}\right.}\right)_{\pi(z)}\right)$. Observe that the semigroup of weights of $T \times T$ in $A$ (resp. $\left.A_{a d}\right)$ is freely generated by $-\chi_{1}, \ldots,-\chi_{r}$ (resp. by the $\left.(-\alpha, \alpha), \alpha \in \Delta\right)$. Because $\pi$ maps $\bar{T}_{z}$ to $\left.\overline{\left(T_{a d}\right.}\right)_{\pi(z)}$, the convex cone generated by the $(-\alpha, \alpha)$ must be contained in the convex cone generated by $-\chi_{1}, \ldots,-\chi_{r}$. Moreover, because restriction of $\pi$ to $T=(T \times T) / \operatorname{diag} T$ is the quotient by $C \times C$, the fraction field of $A_{a d}$ consists in the $(C \times C)$-invariants in the fraction field of $A$. This means that the span of the $(-\alpha, \alpha)$ is the intersection of the span of $\chi_{1}, \ldots, \chi_{r}$ with the span of the character group of $(T \times T) /(C \times C)$, that is, with the span of $\Phi \times \Phi$.

\section{Acknowledgements}

I thank the referee for his careful reading and for his valuable suggestions, especially concerning sections 1.3 and 2.2 .

\section{References}

[BDP] E. Bifet, C. DeConcini, C. Procesi, Cohomology of regular embeddings, Adv. Math. 82 (1990), 1-34.

[Bi] A. Bialynicki-Birula, Some theorems on actions of algebraic groups, Ann. Math. 98 (1973), 480-497.

[BE] D. Barbasch, S. Evens, $K$-orbits on Grassmanians and a PRV conjecture for real groups, J. Algebra 167 (1994), 258-283.

[BB] F. Bien, M. Brion, Automorphisms and local rigidity of regular varieties, Compositio Math. 104 (1996), 1-26.

[Br] M. Brion, Equivariant Chow groups for torus actions, Journal of Transformation Groups 2 (1997), 225-267.

[C] J. Carrell, On the smooth points of a Schubert variety, p. 15-33 in: C. M. S. Conference Proceedings 16, 1995.

[DP1] C. De Concini and C. Procesi, Complete symmetric varieties, in: Invariant Theory (Proceedings, Montecatini 1982), Lecture Note in Math. 996, p. 1-44, Springer-Verlag, New York 1983.

[DP2] C. De Concini and C. Procesi, Complete symmetric varieties II, in: Algebraic Groups and Related Topics, Advanced Studies in Pure Mathematics 6, p. 481-514, Kinokuniya, Tokyo 1985.

[De] V. Deodhar, Some characterizations of Bruhat ordering and determination of the relative Möbius function, Invent. math. 39 (1977), 187-198. 
[EG] D. Edidin and W. Graham, Equivariant Intersection Theory, Invent. math., to appear.

[F] W. Fulton, Intersection theory, Springer-Verlag, New York 1984.

[G] W. Graham, The class of the diagonal in flag bundles, J. Diff. Geom. 45 (1997), 471487.

[H] J. Humphreys, Reflection Groups and Coxeter Groups, Cambridge University Press, Cambridge 1990.

[Kl] S. Kleiman: The transversality of a general translate, Compositio Math. 28 (1974), 287-297.

[Kn] F. Knop, On the Set of Orbits for a Borel Subgroup, Comment. Math. Helv. 70 (1995), 285-309.

[KK1] B. Kostant and S. Kumar, The nil Hecke ring and cohomology of $G / P$ for a Kac-Moody group G, Adv. Math. 62 (1986), 187-237.

[KK2] B. Kostant and S. Kumar, $T$-equivariant $K$-theory of generalized flag varieties, J. Differ. Geom. 32 (1990), 549-603.

[K] S. Kumar, The nil Hecke ring and singularity of Schubert varieties, Invent. math. 123 (1996), 471-506.

[L] V. Lakshmibai, Tangent spaces to Schubert varieties, Math. Res. Lett. 2 (1995), 473477.

[LP] P. Littelmann and C. Procesi, Equivariant Cohomology of Wonderful Compactifications, in: Operator Algebras, Unitary Representations, Enveloping Algebras, and Invariant Theory, Birkhäuser, Basel 1990.

[MS] V. Mehta and V. Srinivas, Normality of Schubert varieties, Amer. J. Math. 109 (1987), 987-989.

[MFK] D. Mumford, J. Fogarty and F. Kirwan, Geometric Invariant Theory, third edition, Springer-Verlag, New York 1994.

[RS] R. W. Richardson and T. A. Springer, The Bruhat order on symmetric varieties, Geometriae Dedicata 35 (1990), 389-436.

[Sp] T. A. Springer, Linear Algebraic Groups, Birkhäuser, Basel 1981.

[St] E. Strickland, A vanishing theorem for group compactifications, Math. Ann. 277 (1987), 165-171.

Michel Brion

Université Grenoble I-CNRS

Institut Fourier UFR de Mathématiques

BP74

F-38402 Saint-Martin d'Hères

France

(Received: June 23, 1997) 